\title{
Thermoelectric Generators (TEGs) and Thermoelectric Coolers (TECs) Modeling and Optimal Operation Points Investigation
}

\author{
Nganyang Paul Bayendang*, Mohamed Tariq Khan, Vipin Balyan
}

Department of Electrical, Electronic and Computer Engineering (DEECE), Cape Peninsula University of Technology (CPUT), Bellville Campus, Cape Town, Western Cape, 7535, South Africa

\begin{tabular}{l} 
A R T I C L E I N F O \\
\hline Article history: \\
Received: 11 September, 2021 \\
Accepted: 05 December, 2021 \\
Online: 10 February, 2022 \\
\hline Keywords: \\
Alternative Energy \\
Energy Efficiency \\
Energy Harvesting \\
Thermoelectric Coolers (TECs) \\
Thermoelectric Generators \\
(TEGs) \\
TEGs and TECs Modeling \\
TEGs/TECs Optimal Operation \\
Thermoelectricity
\end{tabular}

\begin{abstract}
A B S T R A C T
Sustainable energy is gradually becoming the norm today due to greenhouse warming effects; as a result, the quests for different renewable energy sources such as photovoltaic cells as well as energy efficient electrical appliances are becoming popular. Therefore, this article explores the alternative energy case for thermoelectricity with focus on the steadystate mathematics, mixed modelings and simulations of multiple TEGs and TECs modules to study their performance dynamics and to establish their optimal operation points using Matlab and Simulink. The research substantiates that the output current from TEGs or input current to TECs, initially respectively increases the output power of TEGs and the cooling power of TECs, until the current reaches a certain maximum optimal point, after which any further increase in the current, decreases the TEGs' and or TECs' respective output and cooling powers as well as efficiencies, due to Ohmic heating and or entropy change caused by the increasing current. The research main contributions are elaborate easy to understand TEGs/TECs theoretical formulations as well as static and dynamic simulated models in Matlab/Simulink, that can be used initially to dynamically investigate an infinite quantity of TEG and TEC modules connections, be it in series and or in parallel. This is to assist system designers grasp TEGs and TECs theoretical operations better and their limits, when designing energy efficient waste heat recovery (using TEGs)/cooling (using TECs) systems for industrial, residential, commercial and vehicular applications.
\end{abstract}

\section{Introduction}

According to [1], energy security and green economy are becoming paramount today; as a result, the demands for renewable and alternative energy sources such as solar, wind, hydro energy, bio-fuels and fuel cells, as well as energy efficient loads, are on the rise in an effort to ensure energy sustainability and carbon free environment. In this regards, we investigated thermoelectricity as a potential alternative energy for sustainable energy source and loads - that is, as a clean DC power source for low energy lighting/applications and as well to provide clean cooling/heating in various human habitats. Thermoelectricity as reviewed in [2], practically focuses on the Seebeck and Peltier effects. Seebeck effect is basically converting heat to DC electricity and the device that does this is a thermoelectric

\footnotetext{
"Corresponding Author: NP Bayendang, CPUT DEECE, +27765404896, bayendangn@cput.ac.za
}

www.astesj.com

https://dx.doi.org/10.25046/aj070107 generator (TEG). The reversed phenomenon is a Peltier effect which is basically the production of cold from DC electricity and if the direction of current flow changes (swap voltage polarity), heat is also produced and the device that does this is called a thermoelectric cooler (TEC). Therefore, by efficiently applying thermoelectricity prudently, a clean alternative energy source for DC low power applications using TEGs and or energy efficient loads in the forms of heat pumps, air conditioners, refrigerators etc using TECs; can be passably implemented to help sustain some human habitats basic energy consumption such as lighting, cooling and heating; as well as reduce environmental pollution.

As already examined in [2], thermoelectricity lends itself to various applications with focus on how TEGs and TECs can be used respectively as a power source and as a load. Furthermore, studied in [3], is a re-configurable TEG DC-DC converter for maximum TEG energy harvesting in a battery-powered wireless sensors network (WSN). Described in [4], is the analysis and design of a thermoelectric energy harvester (TEH) prototype for 
powering up outdoor sensors and devices. Solar energy was harvested using different TEG arrays in [5] and a theoretical analysis of implementing a re-configurable TEG was researched in [6]. Electronic cooling was investigated in [7] and the findings revealed that the TEC cooling capacity could be increased by increasing its cold side junction temperature and decreasing its temperature difference. A multi-stage TEC module in cascade was examined in [8]; whereas in [9], an extensive mathematical analyses were articulated for TEG and TEC design and materials. A TEG model was developed in [10] for maximum power point tracking but lacks the detailed underlining maths and the parallel TEG combinations was limited to just 2. A comprehensive TEG and TEC models with the detailed maths supporting the TEG and TEC models, were presented respectively in [11] and [12]. In [13], a modeling of TEG using Modelica is asserted but deficient in the comprehensive maths, especially considering modeling infinite multiple TEGs and as well TECs modules - which were not articulated. A parametric ANSYS study of TEG and TEC was presented in [14]; however, the detailed maths and especially for the case for infinite TEG and TEC modules use/ connection, was inadequate. In addition, for large scale TEGs and TECs applications, the following studies were examined. In [15], 600 TEGs with a temperature difference of $\sim 120{ }^{\circ} \mathrm{C}$, were applied to harvest and generate up to $1 \mathrm{~kW}$ of DC power from geothermal heat. It was further indicated a $2 \mathrm{~kW}$ power could be achieved with a higher temperature difference and also the TEG cost is much lower to generate equivalent amount of power than using photovoltaic. However, the study lacks the theoretical details to substantiate it. TEG harvesting of waste thermal energy from household heat sources such as a generator exhaust pipe and a kerosene stove, were performed in [16] and various parameters measurements were made but without detailing the maths to calculate these parameters. Light and heat from the Sun are the most common forms of energy abundant on Earth; as a result, [17] reviewed the possibility of integrating photovoltaic and TEG in a hybrid photovoltaic-TEG system and further examined the efficiency improvement. A 128 TEGs system was assembled in [18] to generate $\sim 684 \mathrm{~W}$ of power from radiation heat transfer at a temperature difference of $\sim 125 \mathrm{~K}$ and with a corresponding power density of $845 \mathrm{~W} / \mathrm{m}^{2}$. Their results further justified that with a greater practical temperature difference of $200 \mathrm{~K}$, the respective generated power and power density of their TEGs system could attain $1.23 \mathrm{~kW}$ and $1.51 \mathrm{~kW} / \mathrm{m}^{2}$. Their TEGs system open circuit voltage, its output power, its power density and its conversion efficiency were investigated in details at different temperature differences; however, the underlining maths was not elaborated. A grid-tied $20 \mathrm{~W}$ TEG experimental model using 24 modules in series with the heat harvested from a waste incinerator, was experimented in a lab and the preliminary and analytical models of the electric output power as a function of specific temperatures, were investigated in [19]. A micro combined cold, heat and power system for a small household with a TEC as the cooler and achieving a cooling power of $26.8 \mathrm{~W}$, was presented in [20]. In [21], a 3D printable TEG device architecture with a high thermocouple density of 190 per $\mathrm{cm}^{2}$ by using a thin substrate as an electrical insulation between the thermoelectric elements, resulted in a high-power output of $47.8 \mu \mathrm{W} / \mathrm{cm}^{2}$ from a $30 \mathrm{~K}$ temperature difference. A stove-powered TEG (SPTEG) was used in [22] to generate power from waste heat released during cooking. They researched series and parallel TEGs connections and the effect of pressure to address low power output due to irregular temperature. Finally, an experimental and a numerical investigations on TECs for comparing air-to-air and air-to-water refrigeration were investigated in [23], with the findings revealing that air-to-water achieves 30-50\% efficiency, compared to air-toair cooling.

These are just a few noted studies; however, lacking in the TEGs/TECs literature are comprehensive details on their maths, modeling and operations when connected in series and also in parallel to increase the output power (in the case of TEG) and the cooling power (in the case of TEC). This article therefore, expands on i) developing and expressing further the theoretical maths covering TEGs and TECs various parameters/modules with focus on the total internal resistance, ii) the modeling of multiple TEGs and TECs modules focusing on their electrical parameters and finally iii) their static and dynamic simulations with focus on the optimal operation points investigation as well as the interpretations thereof. The results are then validated with established published studies and concluding remarks are drawn.

\section{TEGs and TECs Mathematical Analyses and Modeling}

In [9], [11] and [12], the standard static mathematics defining various TEG and TEC parameters as well as their modeling are demonstrated. We developed further and present in the following sections: i) TEGs and TECs maths and ii) the implemented models (based on their maths) using Matlab/Simulink and the simulations of TEG/TEC modules, be it in series and or in parallel connections.

\subsection{TEGs and TECs Steady-state Mathematical Analyses}

The derivations thus far of the TEG and TEC parameters have been based-on the p-n junction thermoelement resistance at the thermocouple level and by extension at the module level as indicated in [9], [11] and [12]. However, in practice, more than one TEG and TEC modules will be needed for more power production and this will take the form of series and or parallel connections; as a result, the electrical resistance will often change. This section redefines the change in $R$ to $R_{t}$ and is articulated next.

\section{(I) TEGs Steady-state Mathematical Analysis}

The following TEG parameters mathematics are developed and presented step-wise for multiple TEGs case as follows:

- Thermoelectric (TE) device p-n junction thermocouple resistance $(r)$

The TE device p-n thermocouple resistance $r$ in ohm is:

$$
r=\rho L / A
$$

with $\rho$ being the TEG/TEC electrical resistivity in $\Omega . \mathrm{m}, L$ is the length in (m) of the TEG/TEC $\mathrm{p}$-n thermocouple and the TEG/TEC $\mathrm{p}$-n thermocouple area is $A$ in metre squared $\left(\mathrm{m}^{2}\right)$.

- TE device (TEG and TEC) module resistance $(R)$ 
The resistance in $(\Omega)$ of a TEG/TEC module is computed as:

$$
R=n r
$$

where $n$ (which differs, could be 100, 127, 199, 255 etc) is a TEG/TEC manufacturer $\mathrm{p}-\mathrm{n}$ thermocouples amount used in a TEG/TEC. The more the $n$, the more powerful is the TEG/TEC.

- $\quad$ TEG/TEC module(s) total resistance $\left(R_{t}\right)$

The total resistance $R_{t}$ in $(\Omega)$ of a TEG/TEC module(s) is simply calculated as:

$$
R_{t}=n \frac{T_{s}}{T_{p}} r=R \frac{T_{s}}{T_{p}}
$$

with $T_{p}$ being the TEGs/TECs (TEG/TEC modules) amount connected in parallel and $T_{s}$ the TEGs/TECs (TEG/TEC modules) amount connected in series. NB: all the TEGs/TECs used in (3), have to be identical model to make sure the $R$ of each TEG/TEC is not vastly different; if not, (3) would be inaccurate.

- $\quad$ TEG(s) output voltage $\left(V_{o}\right)$

The TEG(s) voltage generated in volt, can be derived as:

$$
V_{o}=n S \Delta T-I R_{t}
$$

with $S$ being the TE device Seebeck coefficient in $\mathrm{V} / \mathrm{K}, \Delta T$ $=T_{h}-T_{c}$ the TEG(s) temperature difference in kelvin or ${ }^{\circ} \mathrm{C}$ and the output current of the TEG(s) is $I$ in ampere.

\section{- $\quad$ TEG(s) output current $(I)$}

The TEG(s) generated current $I$ in ampere is deduced as:

$$
I=\frac{n S \Delta T}{R_{L}+R_{t}}
$$

with $R_{L}$ being the resistance of the electrical load connected to the TEG(s) output. NB: more $I$ causes the TEG(s) more Joule heating, which negatively affects the TEGs efficiency.

- $\quad$ TEG(s) hot-side heat absorbed $\left(Q_{h}\right)$

TEG(s) produce DC power when their hot-side is at a high temperature $T_{h}$, during which the TEG(s) becomes hotter and the absorbed heat in watt is $Q_{h}$, given as:

$$
Q_{h}=n\left[\left(S I T_{h}\right)+(K \Delta T)\right]-0.5 I^{2} R_{t}
$$

with $K$ being the TEG(s) thermal conductance in W/K.

- $\quad$ TEG(s) cold-side heat emitted $\left(Q_{c}\right)$

TEG(s) produce DC power when the cold-side of the TEG(s) is at a low temperature $T_{c}$ releasing the heat $Q_{c}$ in watt.

$$
Q_{c}=n\left[\left(S I T_{c}\right)+(K \Delta T)\right]+0.5 I^{2} R_{t}
$$

- $\quad \mathrm{TEG}(\mathrm{s})$ output power $\left(P_{o}\right)$

The TEG(s) modules generated power $P_{o}$ in watt, is found variously as follows:

$$
\begin{aligned}
& P_{o}=Q_{h}-Q_{c} \\
& P_{o}=I V_{o}=n[(S I \Delta T)]-I^{2} R_{t}
\end{aligned}
$$

- TEG(s) electrical/conversion/thermal efficiency $(\eta)$

$\eta$ is the TEG(s) power output $P_{o}$ divided by the TEG(s) hotside heat absorbed $Q_{h} . \eta$ being a performance parameter is:

$$
\eta=P_{o} / Q_{h}
$$

The conversion efficiency details is presented later.

- TEG/TEC Carnot's efficiency $\left(\eta_{c}\right)$

Carnot efficiency is the efficiency determined based-on the temperatures $T_{h}$ and $T_{c}$.

$$
\eta_{c}=\frac{\Delta T}{T_{h}}=\frac{T_{h}-T_{C}}{T_{h}}=1-\frac{T_{C}}{T_{h}}
$$

- $\mathrm{TEG}(\mathrm{s})$ conversion efficiency expression $\left(\eta_{e}\right)$

Simply, $\eta_{e}$ is the raw expression of $\eta$. That is, when equations of $Q_{h}$ and $P_{o}$ (respectively (6) and (8) or (9)) are both substituted in (10).

$$
\eta_{e}=\eta_{c} \frac{\left(n R_{L} / R_{t}\right)}{\left[\left(1+n R_{L} / R_{t}\right)-0.5 \eta_{c}+\left((1 /(2 Z \bar{T}))\left(1+n R_{L} / R_{t}\right)^{2}\left(1+T_{c} / T_{h}\right)\right)\right]}
$$

with $Z \bar{T}$ being the TE device average dimensionless merit figure. NB: $Z$ is the TE device merit figure in per $\mathrm{K}\left(\mathrm{K}^{-1}\right)$ and $\bar{T}=\left(T_{h}+T_{c}\right) / 2$, is the TE device average temperature in $\mathrm{K}$.

- TEG(s) maximum conversion efficiency $\left(\eta_{m}\right)$

$\eta_{m}$ is the efficiency of the TEG(s) at $R_{t} / R_{L}=\sqrt{1+Z \bar{T}}$. The $\eta_{m}$ expression as a function of TEG temperatures and $Z$ is:

$$
\eta_{m}=\eta_{c}\left(\frac{(\sqrt{1+Z \bar{T}})-1)}{\left(\sqrt{1+Z \bar{T}}+\left(T_{c} / T_{h}\right)\right)}\right)
$$

- TEGs maximum power conversion efficiency $\left(\eta_{m p}\right)$

As a function of temperatures and $Z, \eta_{m p}$ is the efficiency of the TEG at its maximum output power $P_{o}$ - that is, at $R_{t}=R_{L}$.

$$
\eta_{m p}=\eta_{c} /\left[2-0.5 \eta_{c}+(2 / Z \bar{T})\left(1+T_{d} / T_{h}\right)\right]
$$

- TEG(s) maximum power output $\left(P o_{\max }\right)$

The TEG(s) maximum transfer of power theoretically happens at $R_{t}=R_{L}$. NB: in practice, $R_{t}=R_{L}$ is hardly ever the case.

$P o_{\max }=(n S \Delta T)^{2}\left(R_{L} / R_{t}\right) / R\left(1+\left(R_{L} / R_{t}\right)\right)^{2}$

- $\mathrm{TEG}(\mathrm{s})$ maximum voltage output $\left(V o_{\max }\right)$

TEG(s) $V o_{\max }$ happens at open circuit, that is when $R_{L}$ is not connected or $R_{L}$ is infinity (extremely large), $I=0 \mathrm{~A}$.

$$
V o_{\max }=n S\left(T_{h}-T_{c}\right)=n S \Delta T
$$

- TEG(s) maximum current output $\left(I_{\max }\right)$

TEG(s) $I_{\text {Max }}$ happens at short circuit - meaning, when the load $R_{L}$ is $0 \Omega$. NB: $R_{t}$ will therefore ideally be the sole resistance.

$$
I_{\text {Max }}=n S \Delta T / R_{t}=n S\left(T_{h}-T_{c}\right) / R_{t}
$$

- $\mathrm{TEG}(\mathrm{s})$ generated current normalized $\left(I_{n}\right)$

$I_{n}$ is the normalized current of the TEG(s) in the range $0 \leq$ $I_{n} \leq 1$. At the TEG(s) maximum transfer of power $\left(R_{t}=R_{L}\right), I_{n}=$ 
0.5. Simply, $I_{n}$ is the TEG(s) generated current divided by the TEG(s) maximum current output. It is calculated as:

$$
I_{n}=\frac{I}{I_{M a x}}=\frac{R_{t}}{R_{t}+R_{L}}
$$

- $\quad$ TEG(s) generated voltage normalized $\left(V_{n}\right)$

$V_{n}$ is the normalized voltage of the TEG(s) ranging from 0 $\leq V_{n} \leq 1$. At the TEG(s) maximum transfer of power (i.e. $R_{L}=R_{t}$ ), $V_{n}=1 / 2 . V_{n}$ is the TEG(s) voltage generated divided by the TEG(s) maximum (ideal) voltage generated. It is given as:

$$
V_{n}=\frac{V_{o}}{V o_{\max }}=\frac{R_{L}}{R_{L}+R_{t}}
$$

- $\quad$ TEG(s) output power normalized $\left(P_{n}\right)$

$P_{n}$ is the normalised TEG(s) power bounded between $0 \leq P_{n}$ $\leq 1 . P_{n}=1$ at the TEG(s) maximum transfer of power $\left(R_{L}=R_{t}\right) . P_{n}$ is the TEG(s) power generated divided by the TEG(s) maximum output power. It is expressed as:

$$
P_{n}=\frac{P_{o}}{P o_{\max }}=\frac{4\left(R_{L} / R_{t}\right)}{\left[\left(R_{L} / R_{t}\right)+1\right]^{2}}
$$

- $\mathrm{TEG}(\mathrm{s})$ conversion efficiency normalized $\left(\eta_{n}\right)$

$\eta_{n}$ is the conversion efficiency of the TEG(s) in the region $0 \leq \eta_{n} \leq 1$. $\eta_{n}$ depends on $R_{t} / R_{L}, T_{c} / T_{h}$ and ZT. $\eta_{n}$ is the conversion efficiency of the TEG(s) divided by the maximum conversion efficiency of the TEG(s), deduced as:

$$
\eta_{n}=\eta / \eta_{m}
$$

- TEG(s) effective Seebeck coefficient $\left(S_{e}\right)$

$S_{e}$ measured in volt/kelvin, is expressed as:

$$
S_{e}=4 P o_{\max } /\left(n I_{\max } \Delta T\right) \quad(\mathrm{V} / \mathrm{K})
$$

- TEG(s) effective electrical resistivity $\left(\rho_{e}\right)$ $\rho_{e}$ measured in ohm metre, is found using:

$$
\rho_{e}=4\left[(A / L) P o_{\max }\right] / n I_{\max }^{2}
$$

- $\mathrm{TEG}(\mathrm{s})$ effective figure of merit $\left(Z_{e}\right)$

$Z_{e}$ measured in per kelvin, is computed as:

$$
Z_{e}=\left[(2 / \bar{T})\left(1+\left(T_{c} / T_{h}\right)\right)\right] /\left[\eta_{c}\left(\left(1 / \eta_{m p}\right)+0.5\right)-2\right] \quad\left(\mathrm{K}^{-1}\right)
$$

- TEG(s)/TEC(s) effective thermal conductivity $\left(k_{e}\right)$

$k_{e}$ measured in watt per metre kelvin, is expressed as:

$$
k_{e}=S_{e}{ }^{2} /\left(\rho_{e} Z_{e}\right) \quad(\mathrm{W} / \mathrm{mK})
$$

TEGs/TECs effective parameters enable researchers to factor in TEGs/TECs system losses using maximum parameters to bridge the theoretical and measured specifications differences [9].

- TEG(s) Heat Flux Density (HFD)

$H F D$ is the amount of heat absorbed per TEGs hot-side surface area (TEGsa) in watt per centimetre square.

$$
H F D=Q_{h} / T E G_{s a} \quad\left(\mathrm{~W} / \mathrm{cm}^{2}\right)
$$

This concludes the TEG(s) modules static mathematical analysis.

\section{(II) TECs Steady-State Mathematical Analysis}

The following TEC parameters mathematics are examined and developed step-wise for multiple TECs case as follows:

- $\quad$ TEC(s) voltage input $\left(V_{\text {in }}\right)$

The TEC(s) applied voltage in volt, is expressed as:

$$
V_{\text {in }}=n\left[S\left(T_{h}-T_{c}\right)\right]+I_{i n} R_{t}
$$

where $I_{\text {in }}$ is the TECs input current from the power supply.

- $\mathrm{TEC}(\mathrm{s})$ input current $\left(I_{\text {in }}\right)$

The TECs input current in ampere is derived as:

$$
I_{\text {in }}=\frac{n S \Delta T}{R_{S}-R_{t}}
$$

where $R_{S}$ is the internal source electrical resistance of the power supply connected to the TECs.

- $\quad$ TEC(s) cold-side heat absorbed $\left(Q_{c}\right)$

TECs create cold when their cold-side is at a low temperature $T_{c}$ to absorb heat and supply a steady cooling power $Q_{c}$ in $\mathrm{W}$.

$$
Q_{c}=n\left[\left(S I_{i n} T_{c}\right)-(K \Delta T)\right]-0.5 I_{i n}^{2} R_{t}
$$

- TEC(s) hot-side heat emitted $\left(Q_{h}\right)$

TECs produce cold when their hot-side is at a high temperature $T_{h}$ emitting the heat $Q_{h}$ in watt.

$$
Q_{h}=n\left[\left(S I_{i n} T_{h}\right)-(K \Delta T)\right]+0.5 I_{i n}^{2} R_{t}
$$

- $\quad$ TEC(s) power input $\left(P_{\text {in }}\right)$

The applied power $P_{\text {in }}$ in watt required to power the TECs, is calculated variously as follows:

$$
\begin{aligned}
& P_{i n}=Q_{h}-Q_{c}=n\left[\left(S I_{i n} \Delta T\right)\right]+I_{i n}^{2} R_{t} \\
& P_{i n}=I_{i n} V_{i n}
\end{aligned}
$$

- TEC(s) coefficient of performance $(\mathrm{CoP})$

This is TECs cooling power $Q_{c}$ divided by its input power $P_{\text {in }}$.

$$
C o P=Q_{d} / P_{\text {in }}
$$

- $\mathrm{TEC}(\mathrm{s})$ CoP Expression $\left(\mathrm{CoP}_{e}\right)$

$\mathrm{CoP}_{e}$ is the raw expression of CoP when the equations of $Q_{c}$ and $P_{\text {in }}$ (respectively (29) and (31) or (32)) are put in (33).

$$
\operatorname{CoP}_{e}=\frac{\left[\left(S I_{i n} T_{C}\right)-(K \Delta T)-\left(0.5 I_{i n}{ }^{2} R_{t} / n\right)\right]}{\left[\left(S I_{i n} \Delta T\right)+\left(I_{i n}{ }^{2} R_{t} / n\right)\right]}
$$

- $\mathrm{TEC}(\mathrm{s})$ current to yield $\mathrm{CoP}\left(I_{c o p}\right)$

$I_{c o p}$ is the TECs input current in (A) needed to attain CoP. 


$$
I_{\text {cop }}=\frac{n S \Delta T}{R_{t}[(\sqrt{1+Z \bar{T}})-1]} \quad \text { (A) }
$$

- TECs maximum $\mathrm{CoP}\left(\mathrm{CoP}_{\max }\right)$

$\mathrm{CoP}_{\max }$ is the TECs maximum $\mathrm{CoP}$ that can be achieved.

$$
C o P_{\max }=\frac{\left[T_{C} / \Delta T\right]\left((\sqrt{1+Z \bar{T}})-\frac{T_{h}}{T_{C}}\right)}{((\sqrt{1+Z \bar{T}})+1)}
$$

- $\mathrm{TEC}(\mathrm{s})$ maximum cooling power current $\left(I c p_{\max }\right)$ $I c p_{\max }$ is TECs current in ampere needed to realise $\max Q_{c}$.

$$
I c p_{\max }=n S T_{d} / R_{t} \quad(\mathrm{~A})
$$

- $\mathrm{TEC}(\mathrm{s}) \mathrm{I}_{\mathrm{cop}}$ maximum cooling power $\left(Q c p_{\max }\right)$ Qcp $\max$ in $(\mathrm{W})$, is TECs maximum $Q_{c}$ attained based-on $I_{c o p}$. $Q_{c p_{\max }}=n\left[\left(S I_{c o p} T_{c}\right)-(K \Delta T)\right]-0.5 I_{c o p}^{2} R_{t} \quad(\mathrm{~W})$

- $\mathrm{TEC}(\mathrm{s})$ maximum temperature difference $\left(\Delta T_{\max }\right)$

TEC(s) $\Delta T_{\max }$ in (K), occurs at maximum $I_{\text {in }}$ and at $Q_{c}=0 \mathrm{~W}$.

$$
\Delta T_{\max }=\left(T_{h}+\frac{1}{Z}\right)-\sqrt{\left(T_{h}+\frac{1}{Z}\right)^{2}-T_{h}^{2}} \quad(\mathrm{~K})
$$

- $\quad$ TEC(s) maximum input current $\left(I_{\max }\right)$

$I_{\max }$ is TEC(s) maximum input current in (A) at $Q_{c}=0 \mathrm{~W}$.

$$
I_{\max }=n S\left(T_{h}-\Delta T_{\max }\right) / R_{t} \quad(\mathrm{~A})
$$

- $\mathrm{TEC}(\mathrm{s})$ maximum input voltage $\left(\operatorname{Vin}_{\max }\right)$

$V_{i n}$ max is the maximum $V_{\text {in }}$ in volt, that produces maximum $\Delta T_{\max }$ when $I_{i n}=I_{\max }, R_{t}=0, T_{c}=0, Q_{c}=0$ and $T_{h}$ is maximum.

$$
\operatorname{Vin}_{\max }=n S T_{h} \quad(\mathrm{~V})
$$

- $\quad \mathrm{TEC}(\mathrm{s})$ maximum cooling power $\left(Q c_{\max }\right)$

$Q c_{\max }$ is the maximum absorbable heat or cooling power in watt, at $I_{\text {in }}=I_{\max }$ and $\Delta \mathrm{T}=0^{\circ} \mathrm{C}$.

$$
Q c_{\max }=(n S)^{2}\left(T_{h}^{2}-\Delta T_{\max }^{2}\right) / 2 R_{t}
$$

- TEC(s) input current normalized $\left(\operatorname{Iin}_{n}\right)$

TEC(s) $\operatorname{Iin}_{n}$ is $I_{\text {cop }}$ divided by $I_{\max }$.

$$
\operatorname{Iin}_{n}=I_{c o p} / I_{\max }
$$

- $\mathrm{TEC}(\mathrm{s})$ input voltage normalized $\left(\operatorname{Vin}_{n}\right)$

TEC(s) $\operatorname{Vin}_{n}$ is $V_{\text {in }}$ divided by $\operatorname{Vin}_{\max }$.

$$
\operatorname{Vin}_{n}=V_{\text {in }} / \operatorname{Vin}_{\max }
$$

- $\quad$ TEC(s) cooling power normalized $\left(Q c_{n}\right)$

TEC(s) $Q c_{n}$ is $Q_{c}$ divided by $Q c_{\max }$.

$$
Q c_{n}=Q d Q c_{\max }
$$

- $\quad \mathrm{TEC}(\mathrm{s}) \mathrm{CoP}$ normalized $\left(\mathrm{CoP}_{n}\right)$

TEC(s) $C_{o} P_{n}$ is $C o P$ divided by $C o P_{\max }$.

$$
\mathrm{CoP}_{n}=\mathrm{CoP} / \mathrm{CoP} P_{\max }
$$

- TEC(s) normalized temperature difference $\left(\Delta T_{n}\right)$

TECs $\Delta T_{n}$, is $\Delta T$ divided by $\Delta T_{\max }$ and it is expressed as:

$$
\Delta T_{n}=\Delta T / \Delta T_{\max }
$$

Normalized parameters give dimensionless parameters.

- TEC(s) effective Seebeck coefficient $\left(S_{e}\right)$

TECs $S_{e}$ measured in $\mathrm{VK}^{-1}$, is defined as:

$$
S_{e}=2 Q c_{\max } /\left[n I_{\max }\left(T_{h}+\Delta T_{\max }\right)\right] \quad(\mathrm{V} / \mathrm{K})
$$

- TEC(s) effective electrical resistivity $\left(\rho_{e}\right)$

TECs $\rho_{e}$ measured in ohm metre, is written as:

$$
\rho_{e}=A S_{e}\left(T_{h}-\Delta T_{\max }\right) / L I_{\max } \quad(\Omega . \mathrm{m})
$$

- $\quad$ TEC(s) effective figure of merit $\left(Z_{e}\right)$

TECs $Z_{e}$ measured in per kelvin, is given as:

$$
Z_{e}=2 \Delta T_{\max } /\left(T_{h}-\Delta T_{\max }\right)^{2} \quad\left(\mathrm{~K}^{-1}\right)
$$

- $\quad$ TEC(s) midpoint current $\left(I_{m i d}\right)$

$I_{\text {mid }}$ measured in ampere, is the mean of Icp $p_{\max }$ and $I_{c o p}$.

$$
I_{\text {mid }}=0.5\left(I c p_{\max }+I_{c o p}\right)
$$

- $\quad$ TEC(s) midpoint cooling power $\left(Q c_{m i d}\right)$

$Q c_{\text {mid }}$ measured in watt, is expressed as:

$$
Q c_{\text {mid }}=n\left[\left(S I_{\text {mid }} T_{c}\right)-(K \Delta T)\right]-0.5 I_{\text {mid }}^{2} R_{t}
$$

- $\quad \mathrm{TEC}(\mathrm{s})$ midpoint input power $\left(\operatorname{Pin}_{\text {mid }}\right)$

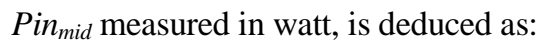

$$
\operatorname{Pin}_{\text {mid }}=n\left[\left(S I_{\text {mid }} \Delta T\right)\right]+I_{\text {mid }}^{2} R_{t}
$$

- $\quad \mathrm{TEC}(\mathrm{s})$ midpoint $\mathrm{CoP}\left(\mathrm{CoP}_{\text {mid }}\right)$

$\mathrm{CoP}_{\text {mid }}$ is computed as:

$$
C o P_{\text {mid }}=Q c_{\text {mid }} / \text { Pin }_{\text {mid }}
$$

Midpoint parameters ascertain safer optimal TECs design.

- $\quad$ TEC(s) cold flux density (CFD)

$C F D$ is the cold amount produced (heat absorbed) per TECs cold-side surface area (TECsa) in W/ $\mathrm{cm}^{2}$. It is computed as:

$$
C F D=Q_{d} / T E C s a \quad\left(\mathrm{~W} / \mathrm{cm}^{2}\right)
$$

\subsection{TEGs and TECs Modelling and Simulations}

Covered in Section 2.1., are the TEGs and TECs parameters of interests - which were extensively expressed mathematically with emphasis/basis on the total internal resistance $R_{t}$ - which was derived and the regular TEG/TEC equations re-expressed basedon $R_{t}$ to now cover TEG(s)/TEC(s). The above equations are herein further modeled in Matlab and Simulink, to institute the TEGs and TECs models that can now be utilized to simulate and investigate many connected TEGs and TECs optimal performance. 
Exemplified in Figures 1a and 1b, are the TEGs static and dynamic simulated model GUIs, from which the TEGs parameters expressed in Section 2.1.I, can all be statically and dynamically configured for an infinite amount of TEGs connections and then simulated to obtain the TEG(s) optimum operation points. Figures $1 \mathrm{c}$ and $1 \mathrm{~d}$ zoom-in on the TEGs internal modeling. Figure 1e expands on the TEGs $R_{t}$ modeling - this must be matched to the load resistance $R_{L}$
- which can be changed before or while the simulation is running to match the TEGs $R_{t}$ for maximum power transfer simulation. Figure 2 exemplifies the TECs simulator user interface. Also, multiple TECs combinations in $T_{s}$ and $T_{p}$ and the various parameters presented in Section 2.1.II, can be optimally simulated. Likewise, maximum power will be transferred also from the DC power supply to the TECs by matching its $R_{t}$ to $R_{s}$.

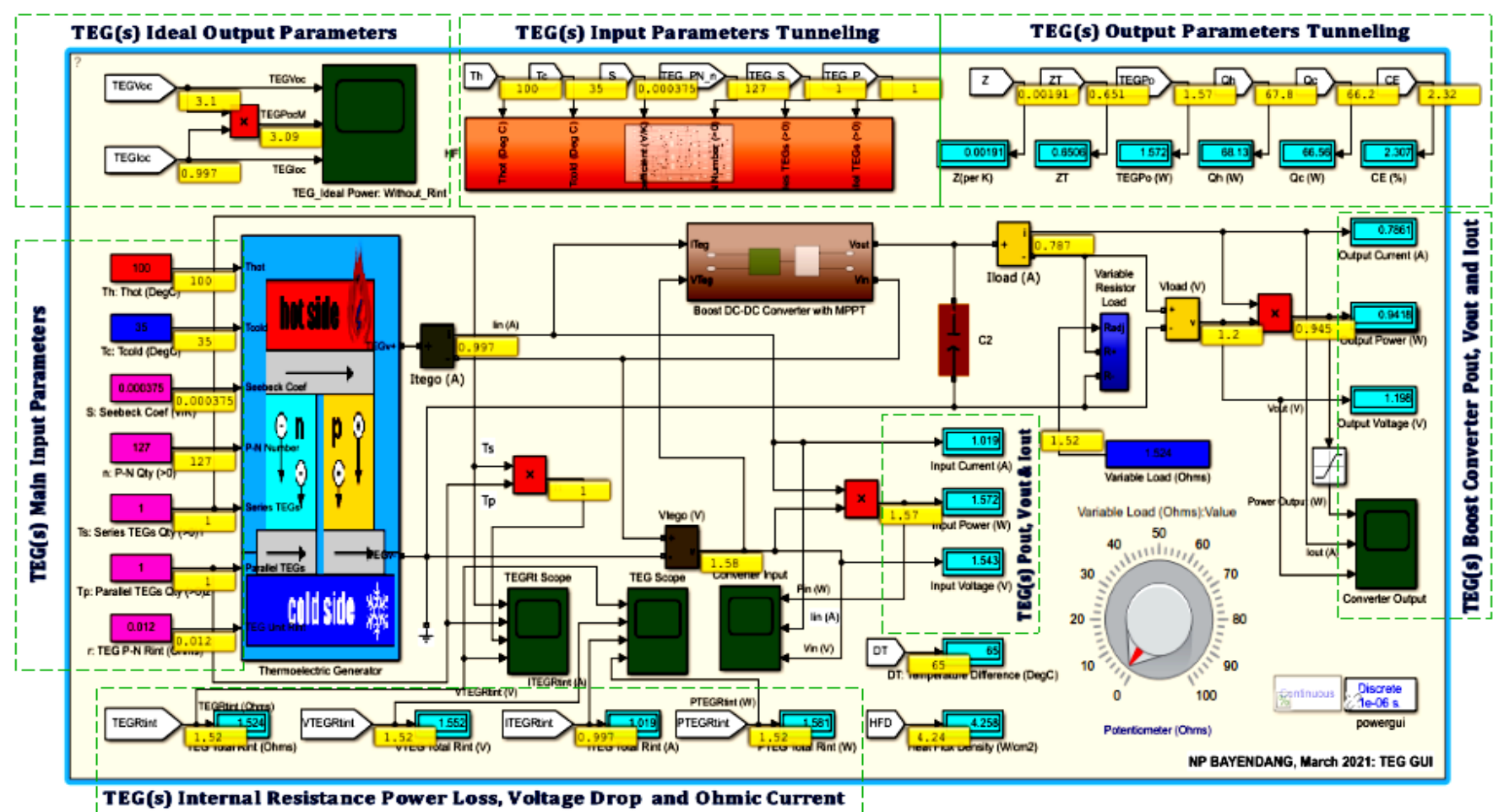

Figure 1a: TEG(s) static simulator user's interface - shows the steady-state simulation with all the input parameters fixed (though can change) over-time

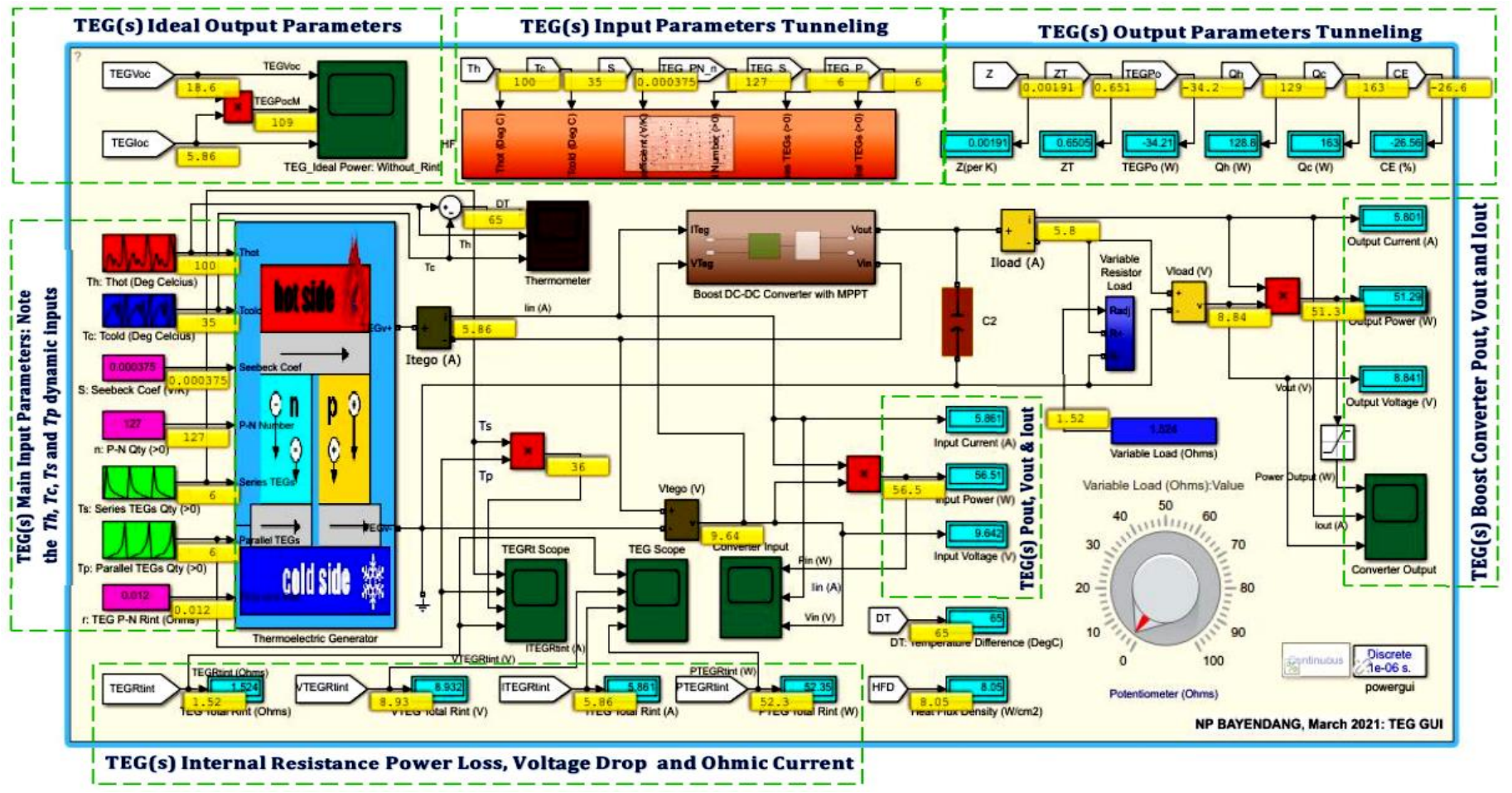

Figure 1b: TEG(s) dynamic simulator user's interface - shows the transient simulation with the $T_{h}, T_{c}, T_{s}$ and $T_{p}$ input parameters auto changing with time 


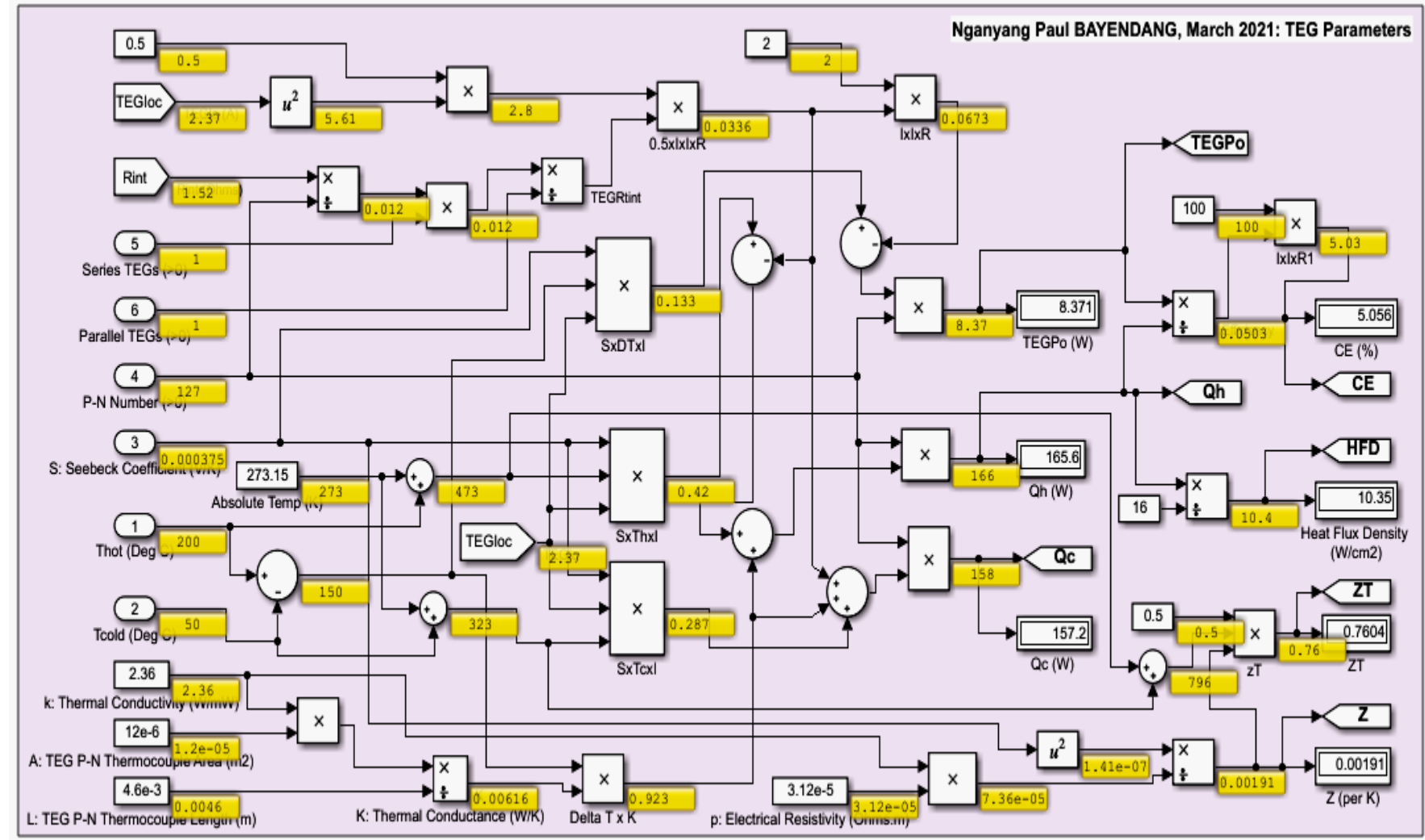

Figure 1c: TEG(s) modeling and simulation - TEG(s) parameters

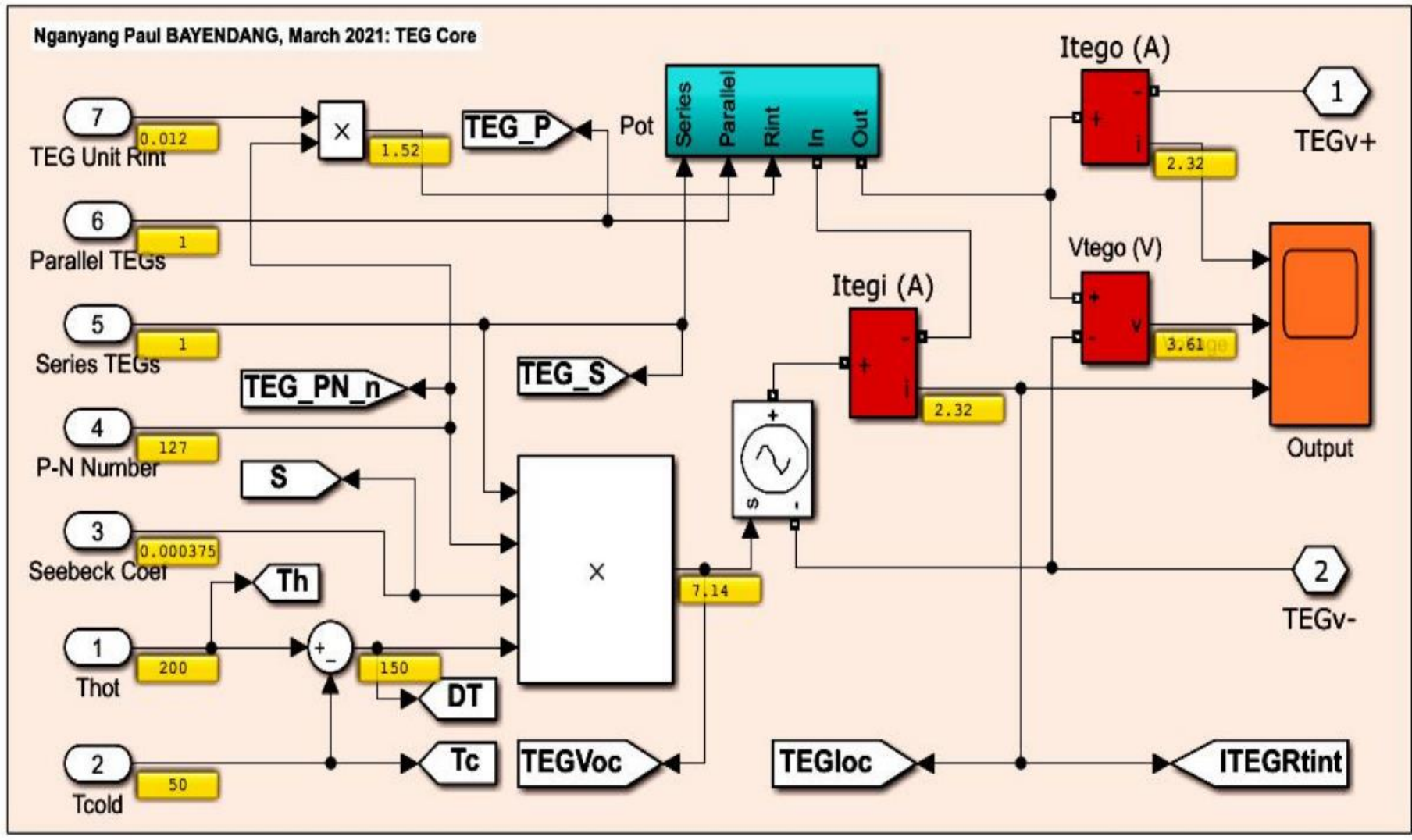

Figure 1d: TEG(s) modeling and simulation - TEG(s) engine 


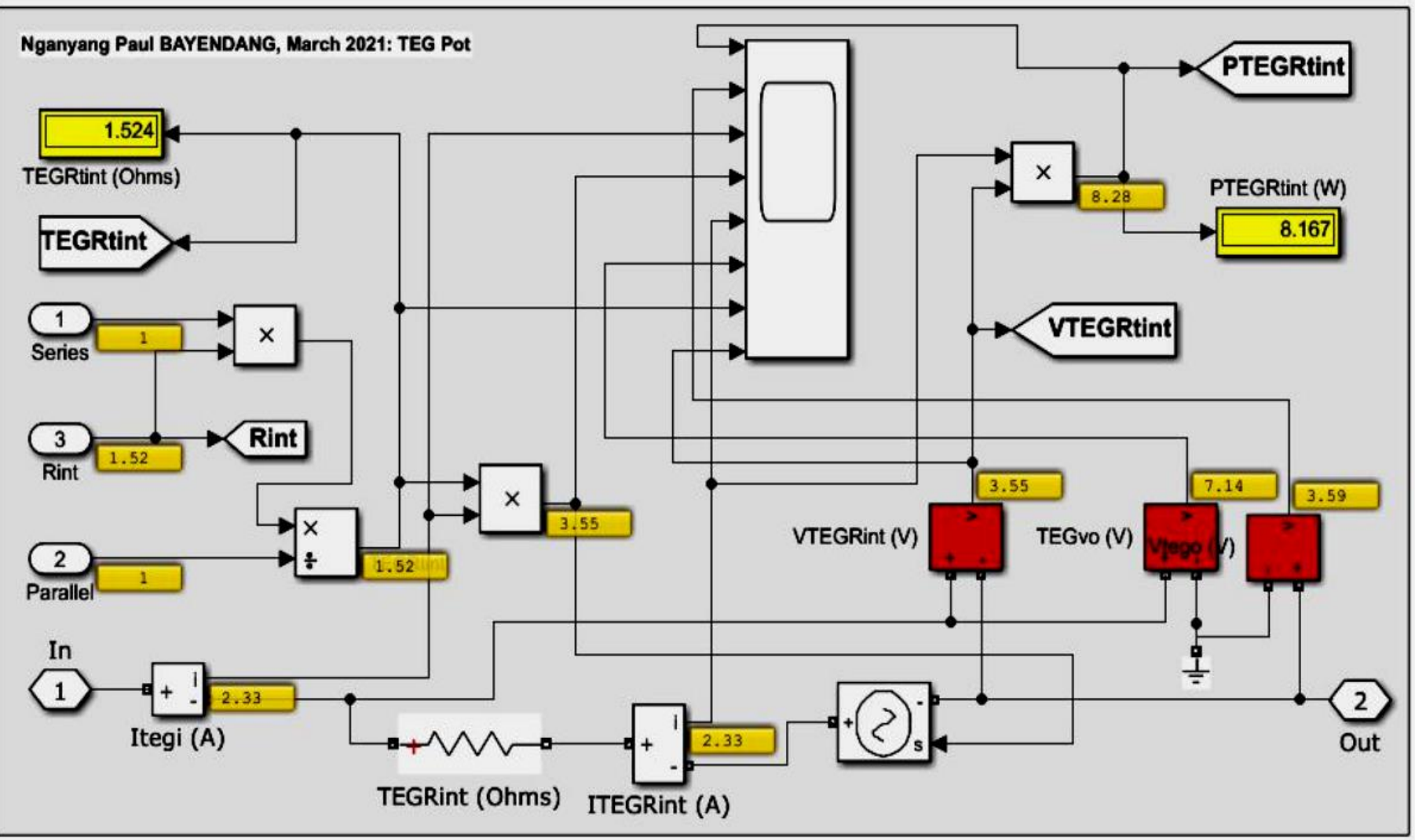

Figure 1e: TEG(s) modeling and simulation - TEG(s) automatic internal source total electrical resistance $R_{t}$

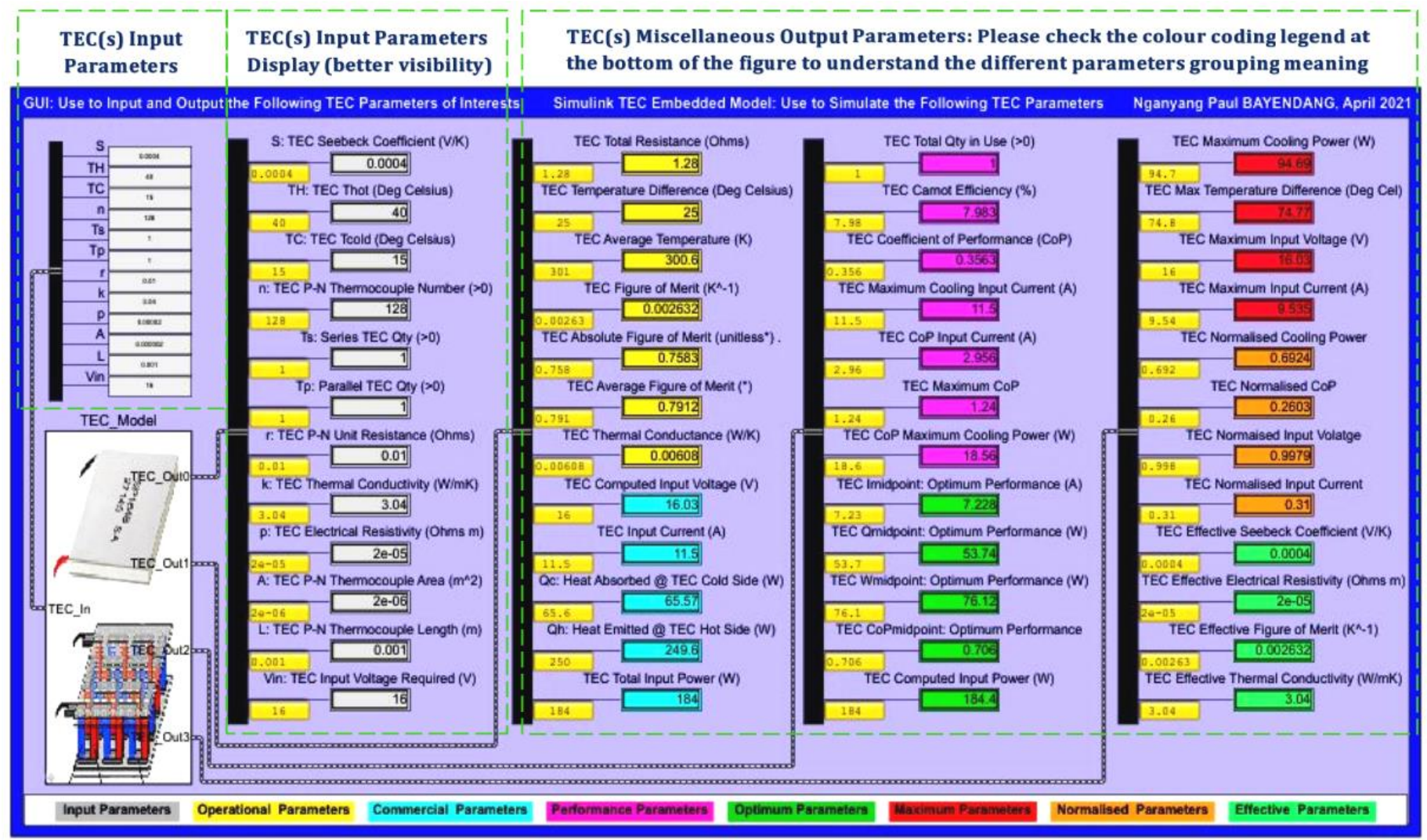

Figure 2: TEC(s) simulator - simulates TECs various parameters by inputting a TEC specific data sheet parameters and calculates its theoretical outputs 


\section{TEGs and TECs Simulations Results}

The TEGs and TECs simulations results are presented in three parts as follows, the i) TEGs parameters static simulation results ii) TECs parameters static simulation results and iii) TEGs parameters dynamic simulation results. Understanding these parameters operation is very paramount; otherwise, doing the physical design would just be a matter of taking chances and hoping for the best - which is sometimes the case, as most designers have reported very bad design results, likely from not understanding TE devices dynamic operations and limitations. The results from investigating the TEG(s) and TEC(s) parameters optimal operation points are discussed in details in Section 4.

\subsection{TEGs Parameters Static Simulation Results}

Figures $3-6$ expound the TEGs parameters simulated to determine their optimal operation points - marked in green.

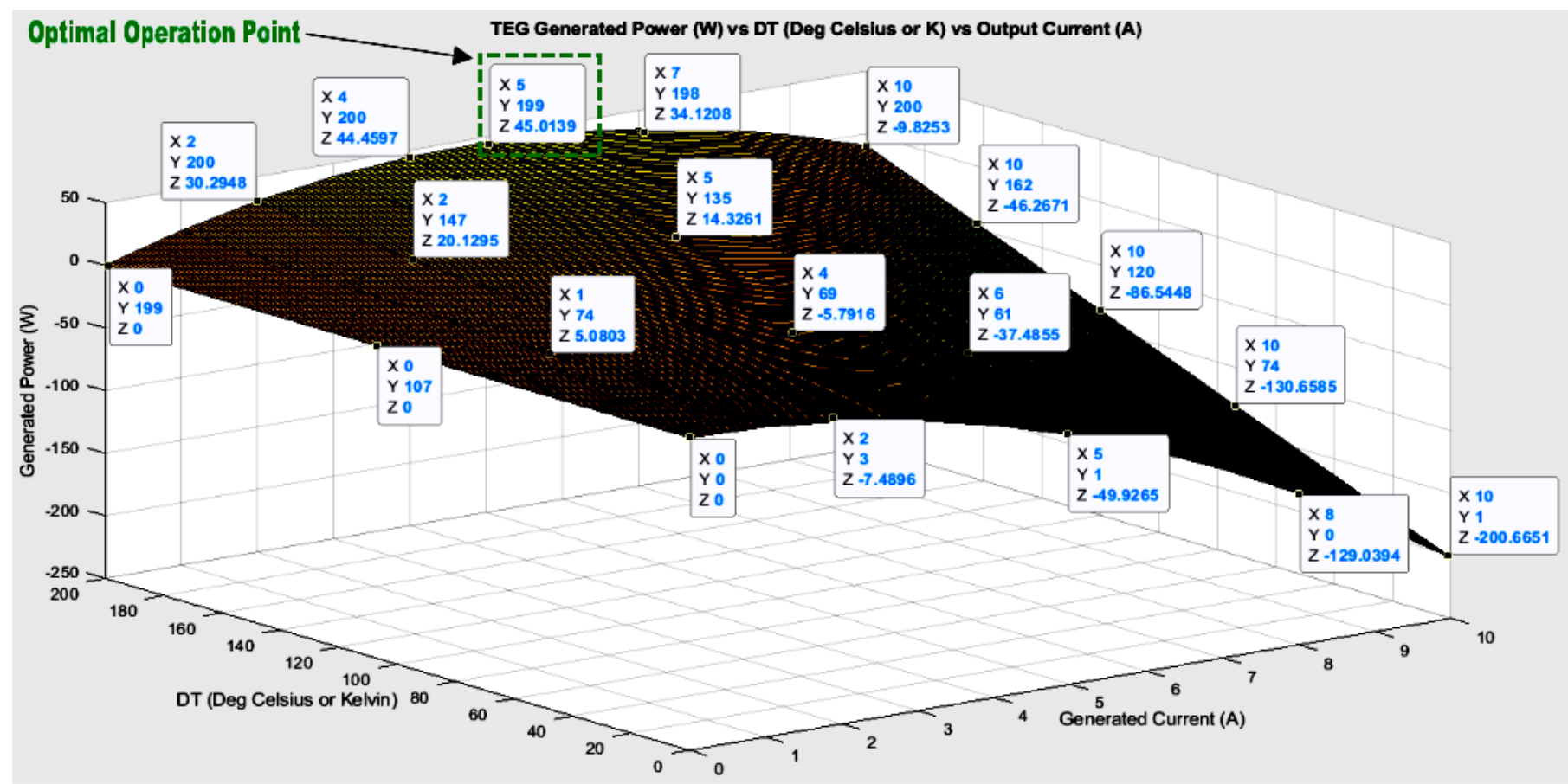

Figure 3: TEG power output $P_{o}(\mathrm{~W})$ vs temperature difference $\Delta \mathrm{T}\left({ }^{\circ} \mathrm{C}\right)$ vs current output $I(\mathrm{~A})$

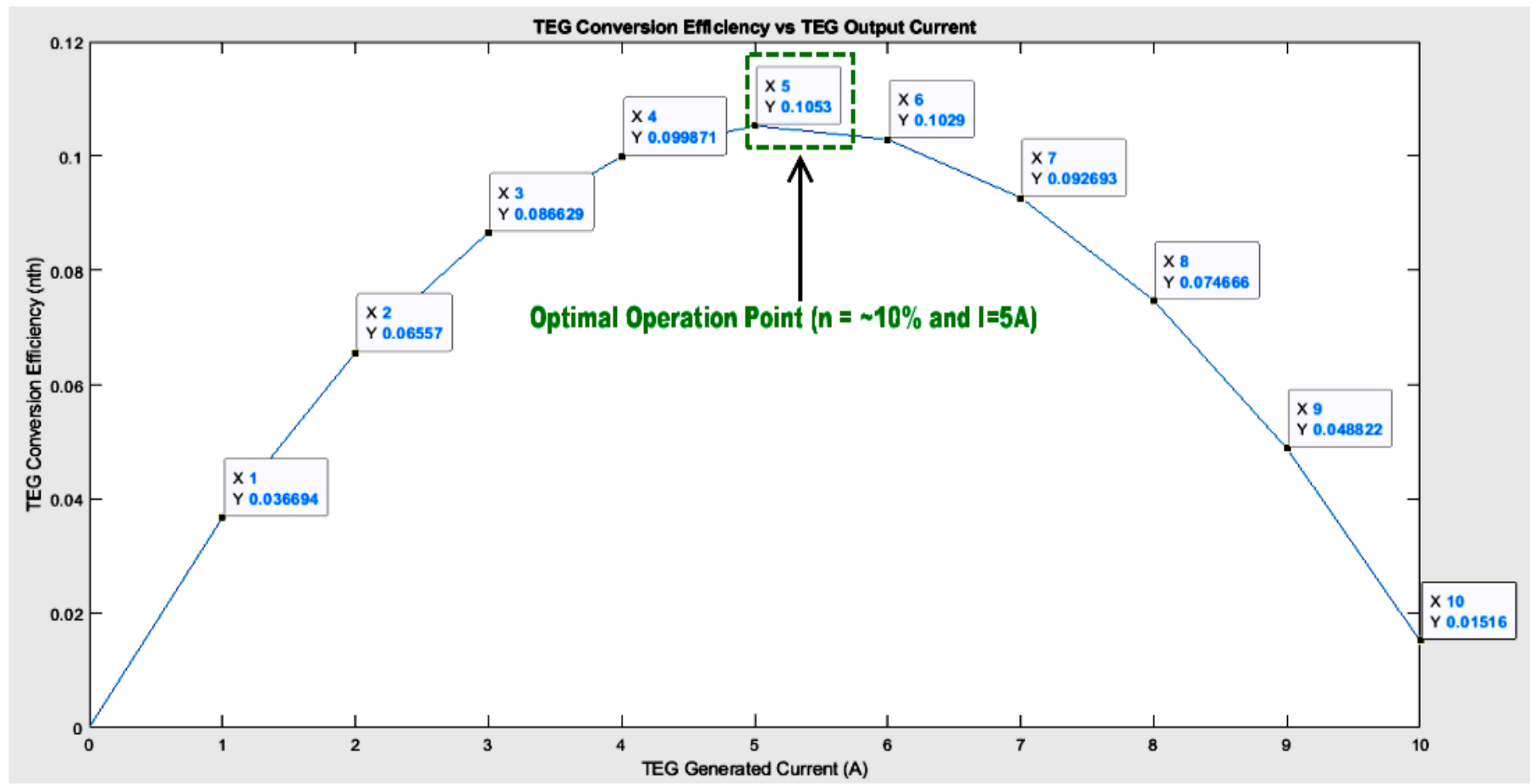

Figure 4: TEG conversion efficiency $\eta$ vs current output $I$ (A) 
TEG Generated Power (W) vs Output Current (A) vs PN Thermocouple Resistance (Ohms)

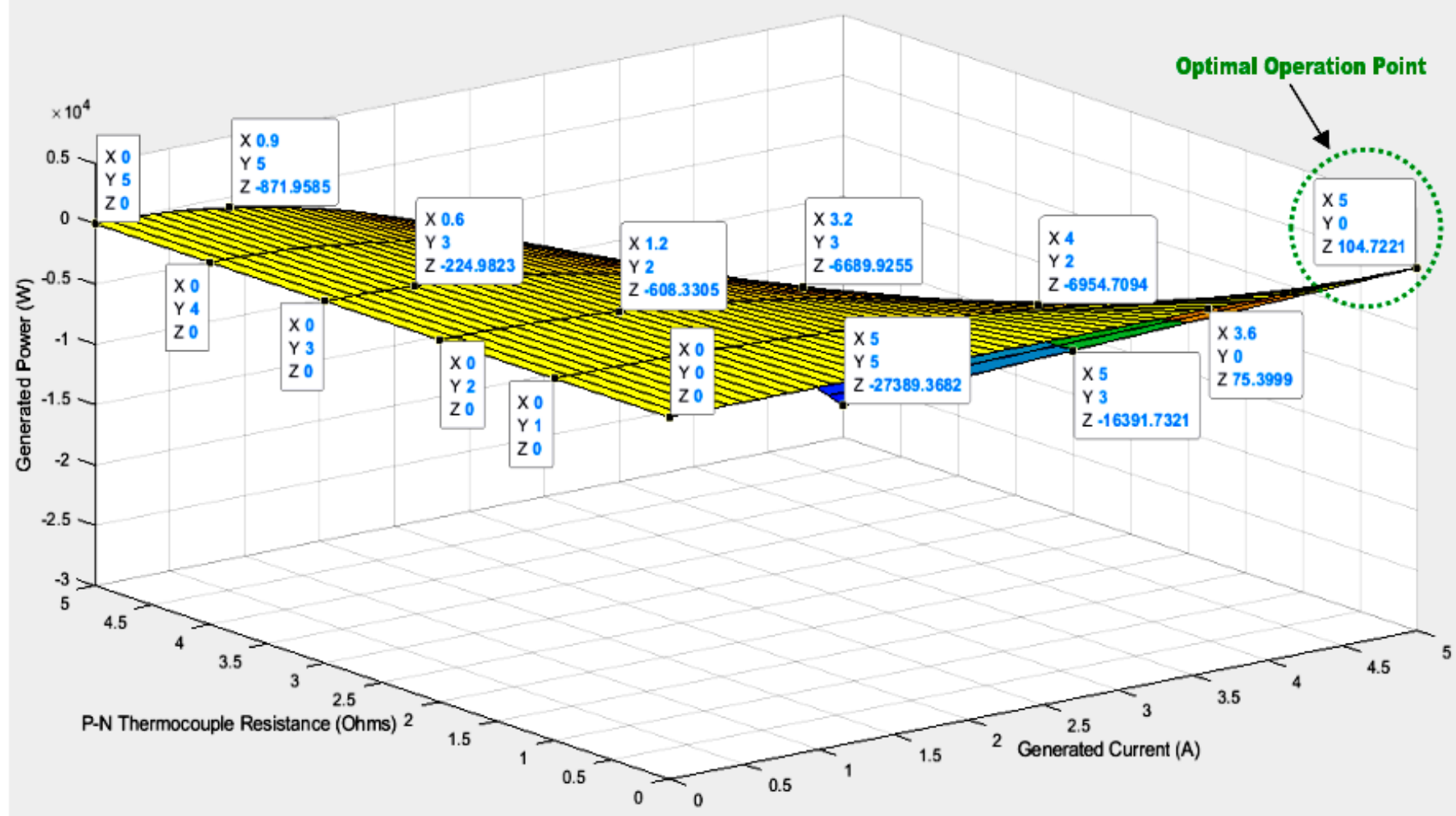

Figure 5: TEG power output $P_{o}(\mathrm{~W})$ vs $r$ or $R$ or $R_{t}(\Omega)$ vs current output $I$ (A)

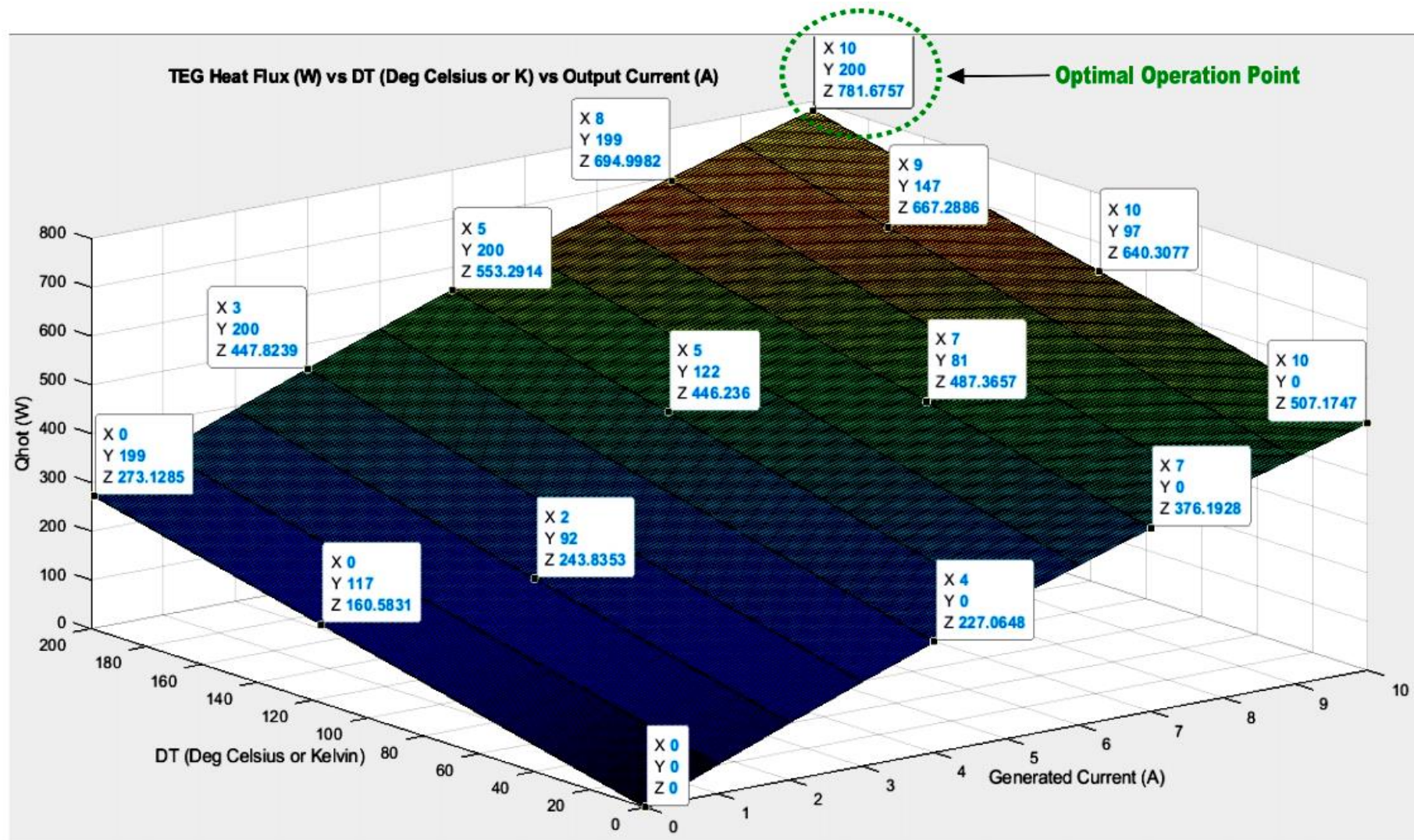

Figure 6: TEG absorbed heat $Q_{h}(\mathrm{~W})$ vs temperature difference $\Delta \mathrm{T}\left({ }^{\circ} \mathrm{C}\right)$ vs output current $I(\mathrm{~A})$ 


\subsection{TECs Parameters Static Simulation Results}

TECs parameters are simulated in Figures 7 - 10 to determine their possible optimal operation points - shown highlighted in red.

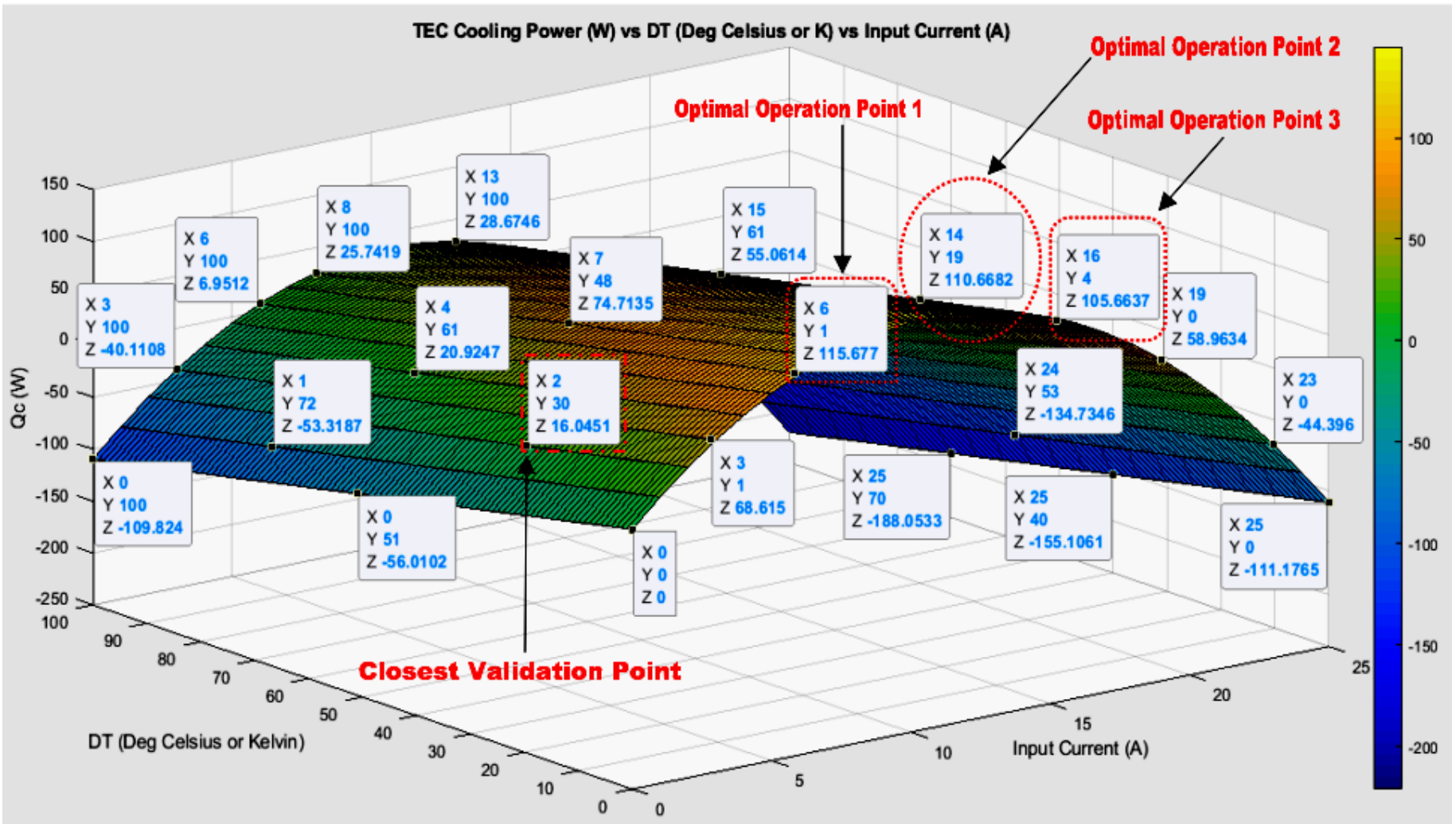

Figure 7: TEC cooling power or heat absorbed $Q_{c}(\mathrm{~W})$ vs temperature difference $\Delta \mathrm{T}\left({ }^{\circ} \mathrm{C}\right)$ vs input current $I_{i n}(\mathrm{~A})$

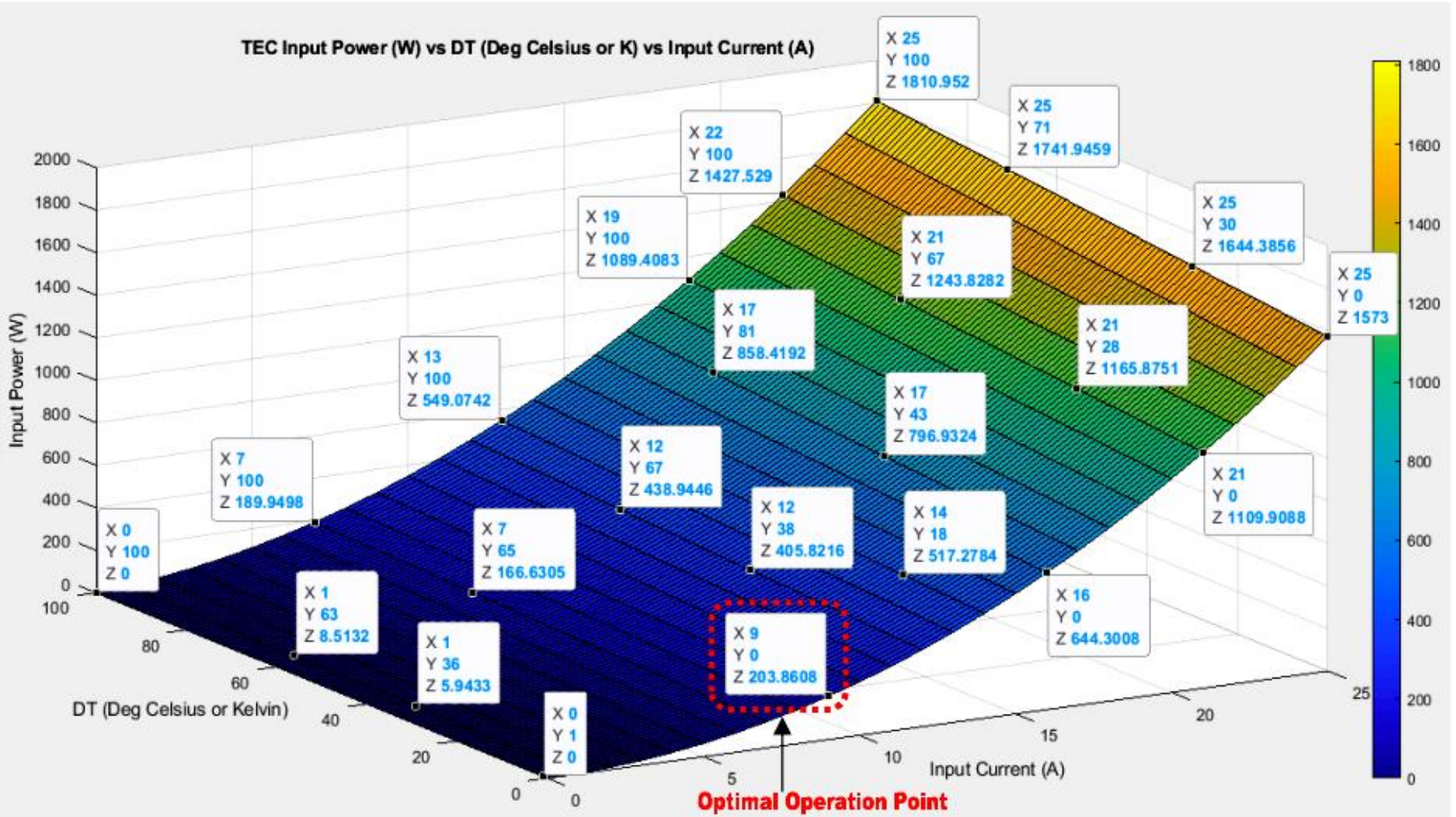

Figure 8: TEC input power $P_{\text {in }}(\mathrm{W})$ vs temperature difference $\Delta \mathrm{T}\left({ }^{\circ} \mathrm{C}\right)$ vs input current $I_{\text {in }}(\mathrm{A})$ 


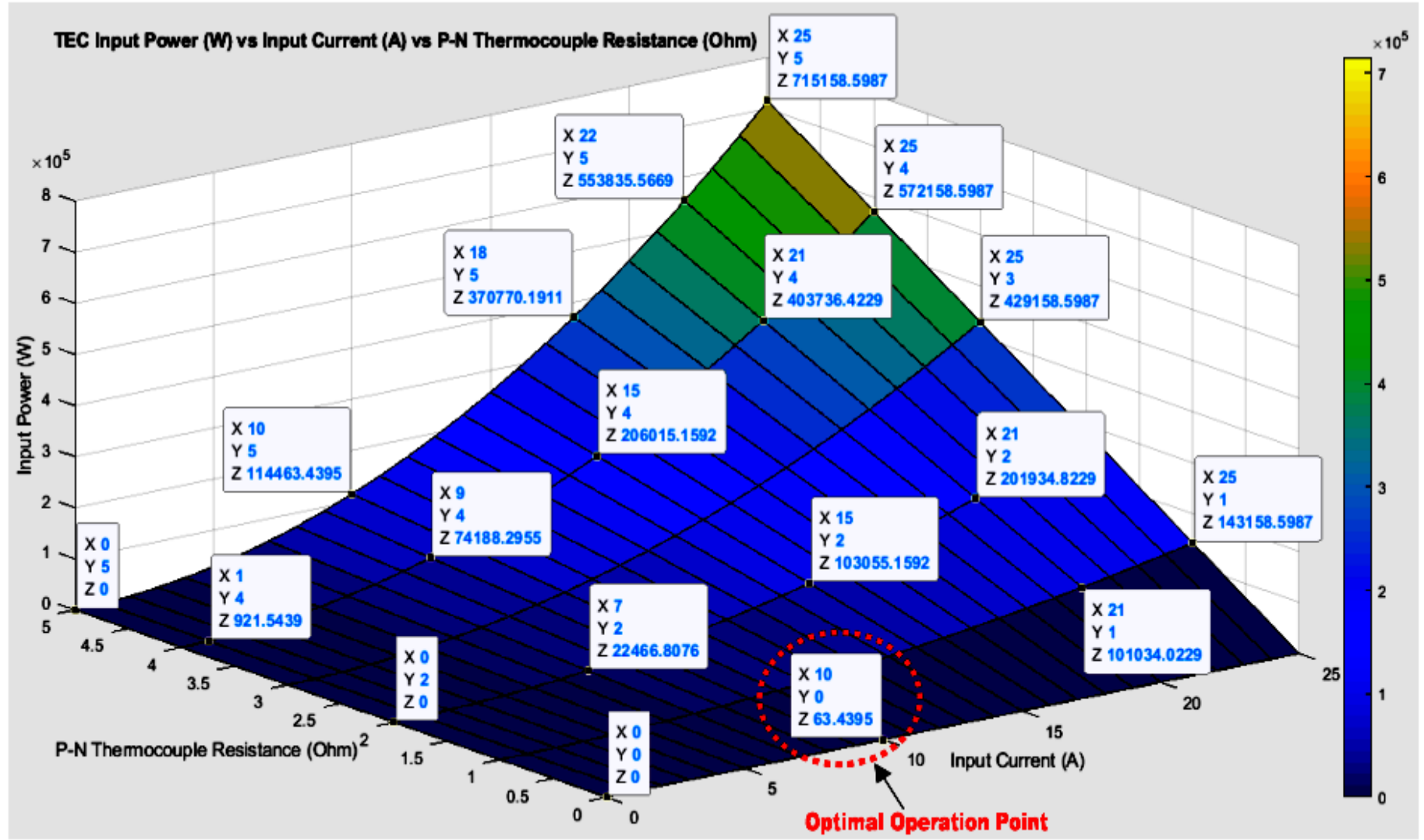

Figure 9: TEC input power $P_{\text {in }}(\mathrm{W})$ vs internal resistance $r$ or $R$ or $R_{t}(\Omega)$ vs input current $I_{\text {in }}$ (A)

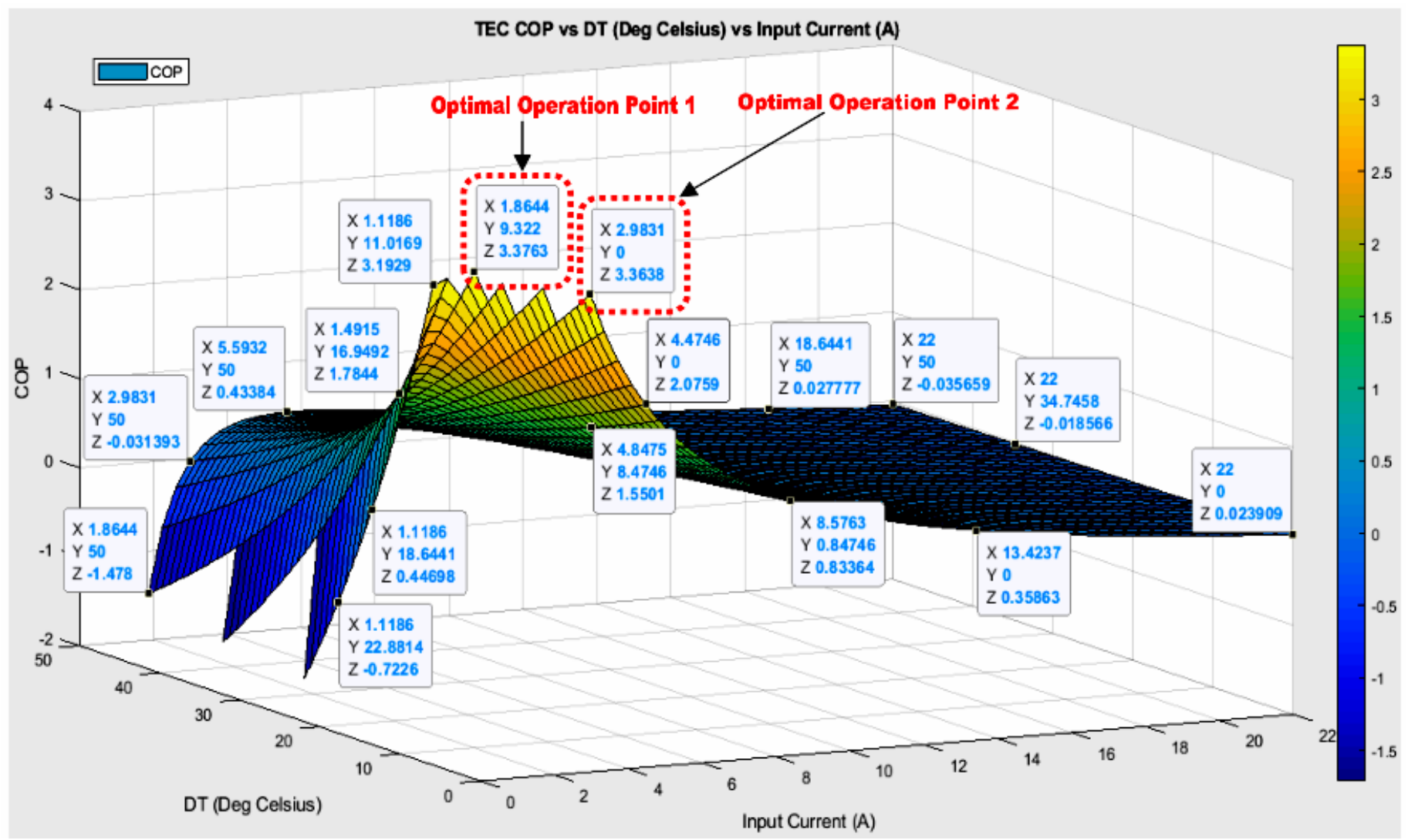

Figure 10: TEC coefficient of performance $\operatorname{CoP}(\%)$ vs temperature difference $\Delta T\left({ }^{\circ} \mathrm{C}\right)$ vs input current $I_{i n}(\mathrm{~A})$ 


\subsection{TEGs Parameters Dynamic Simulation Results}

TEG modules temperatures, its series and parallel connections dynamic simulation results are depicted in Figures 11a - 11f.

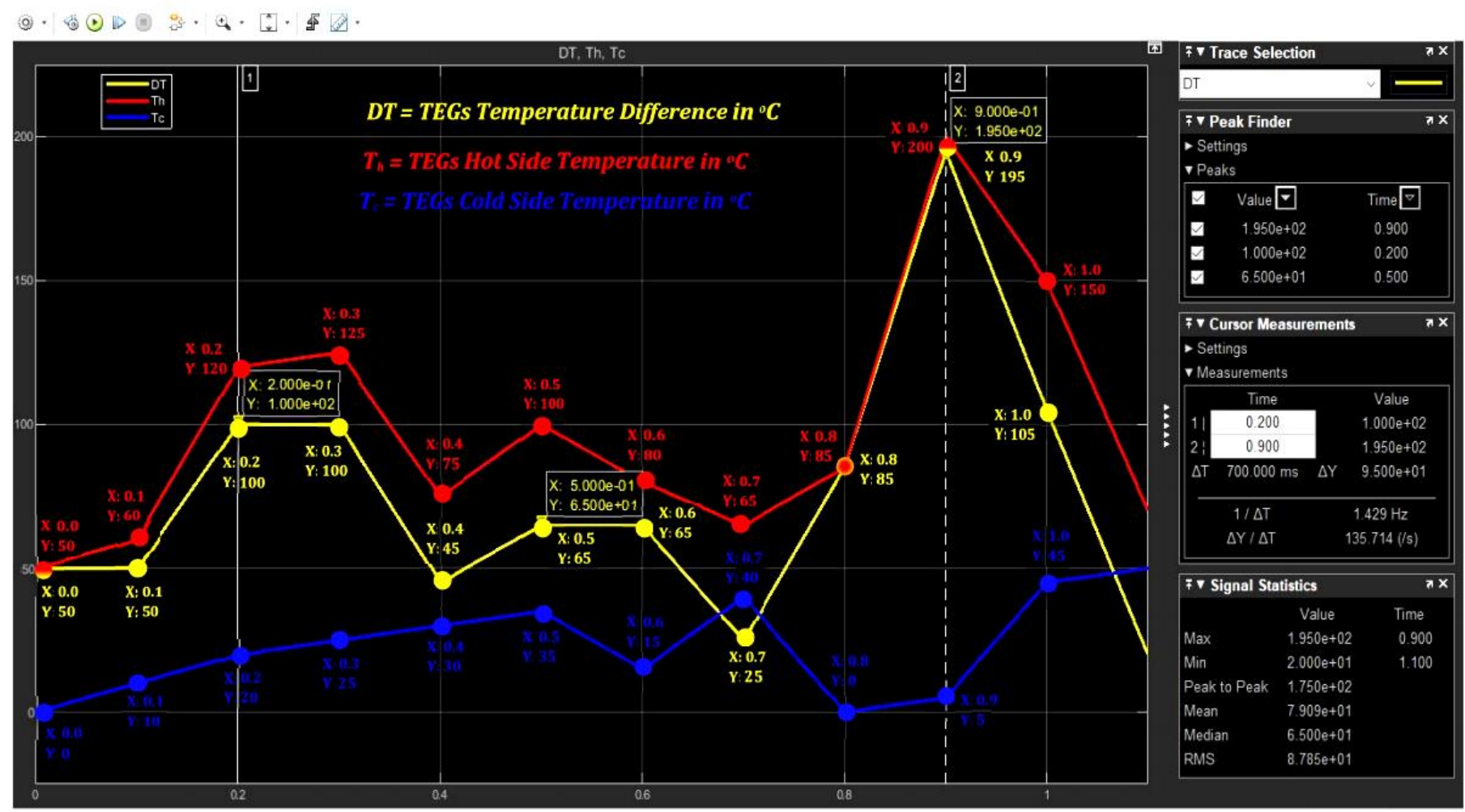

Figure 11a: 36 TEGs hot $\left(T_{h}\right)$ and cold $\left(T_{c}\right)$ temperatures as well as temperature difference $(D T)$ dynamics - temperature changes as simulation progresses

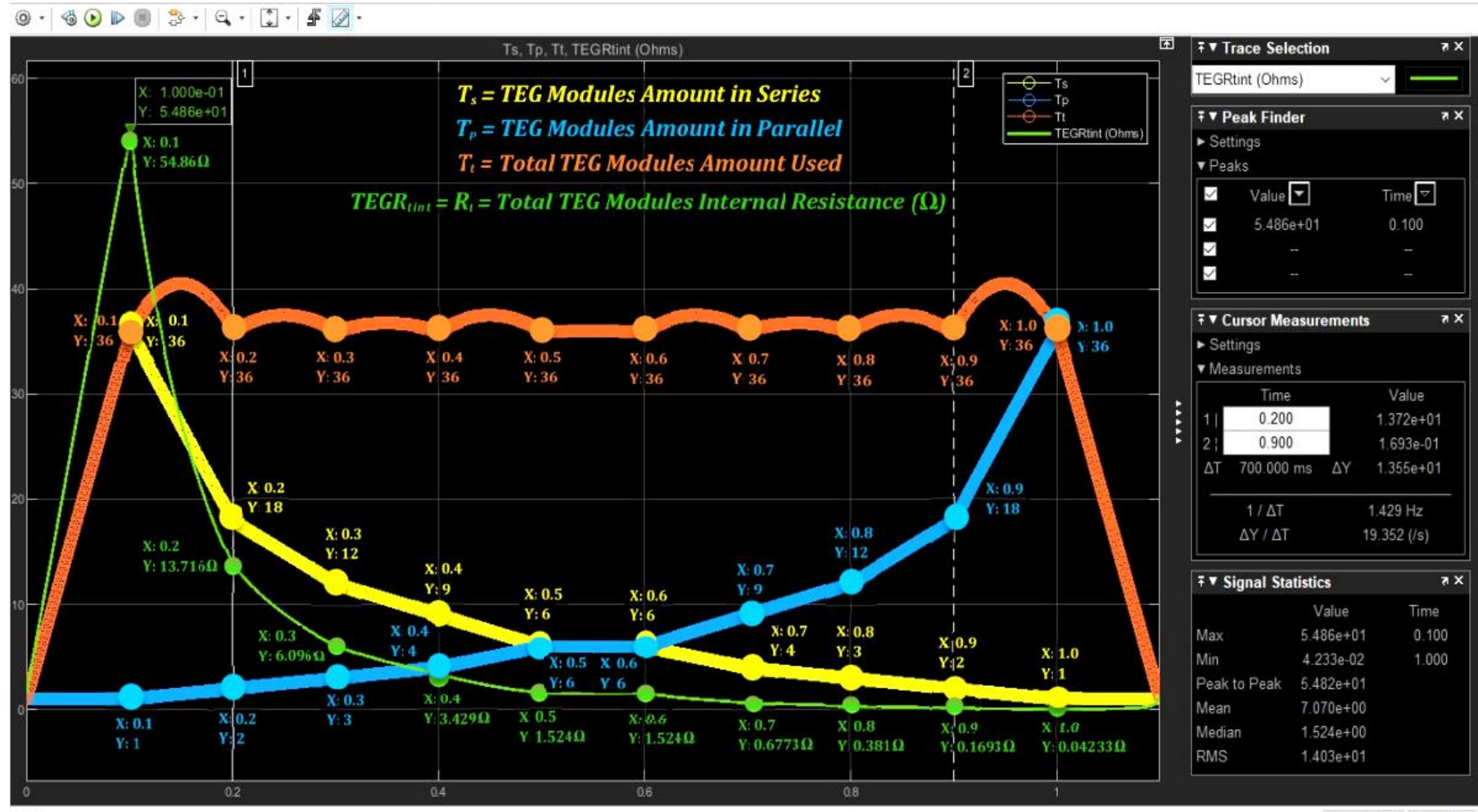

Figure 11b: TEGs in series $\left(T_{s}\right)$, parallel $\left(T_{p}\right)$ and total internal resistance $\left(R_{t}\right)$ dynamics -36 TEG modules simulated in 10 different auto reconfiguration 


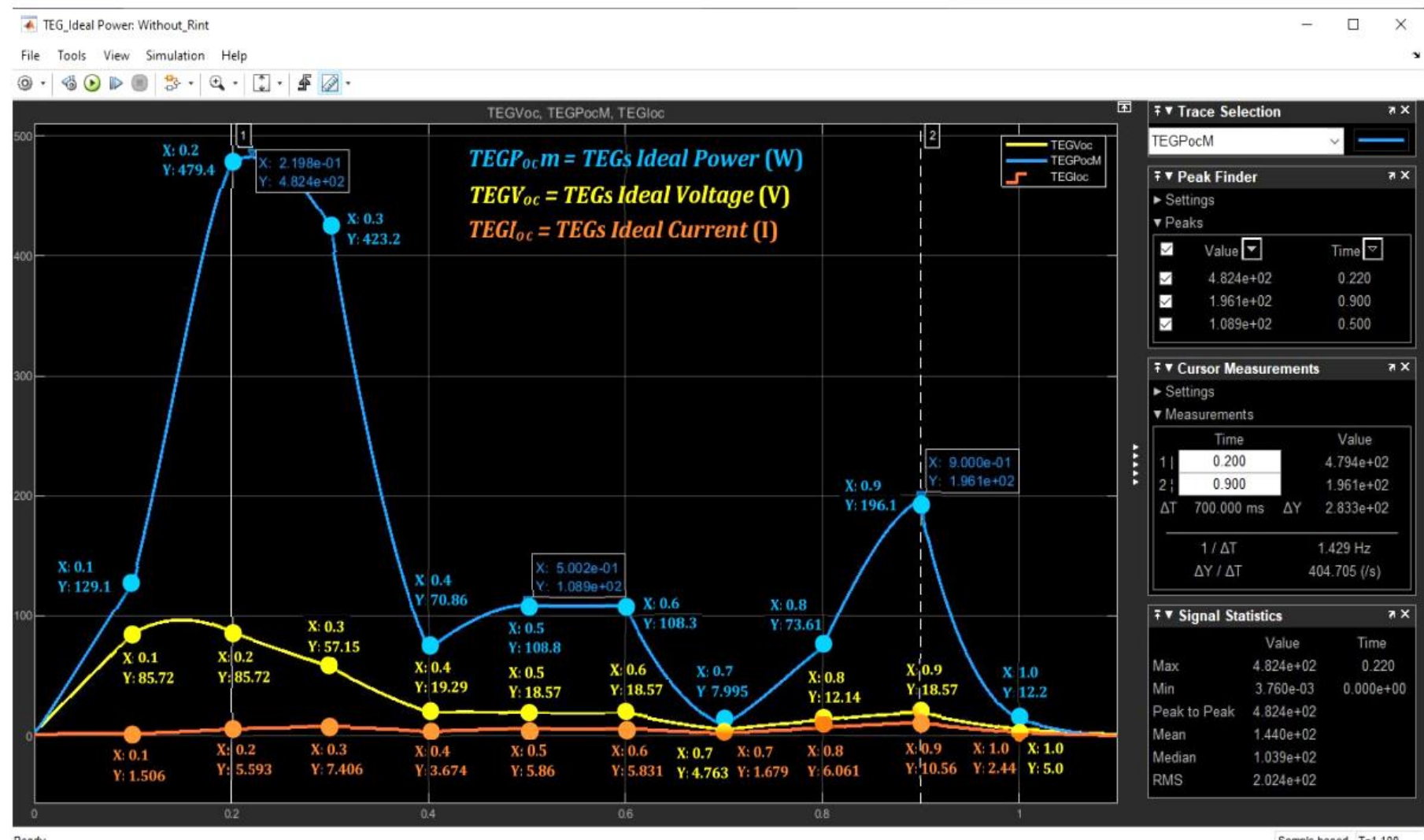

Figure 11c: 36 TEGs ideal output power, voltage and current dynamics; as TEGs temperatures and its 10 configurations change as simulation progresses

A. TEG Scope

File Tools View simulation Help

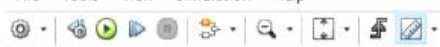

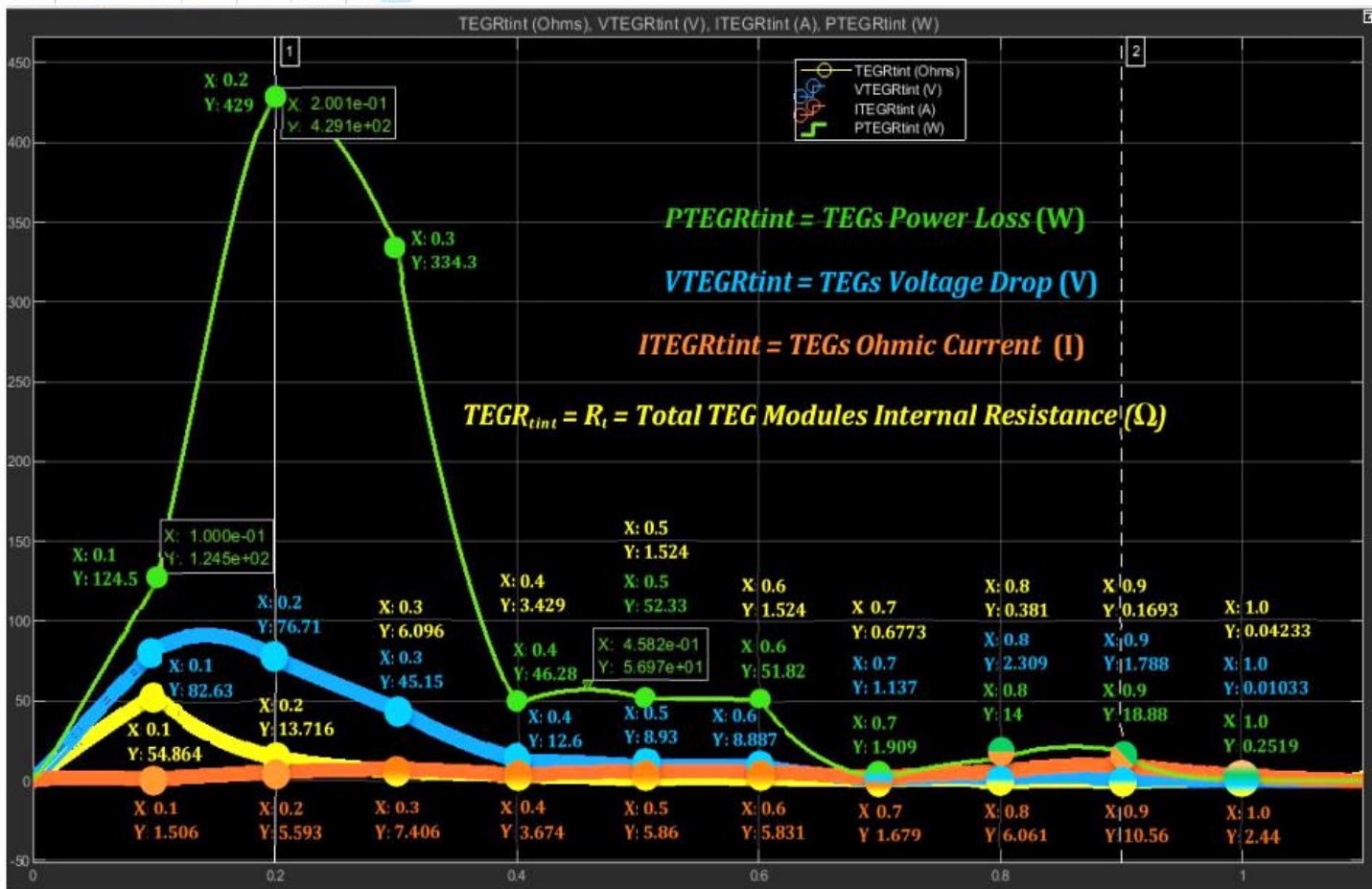

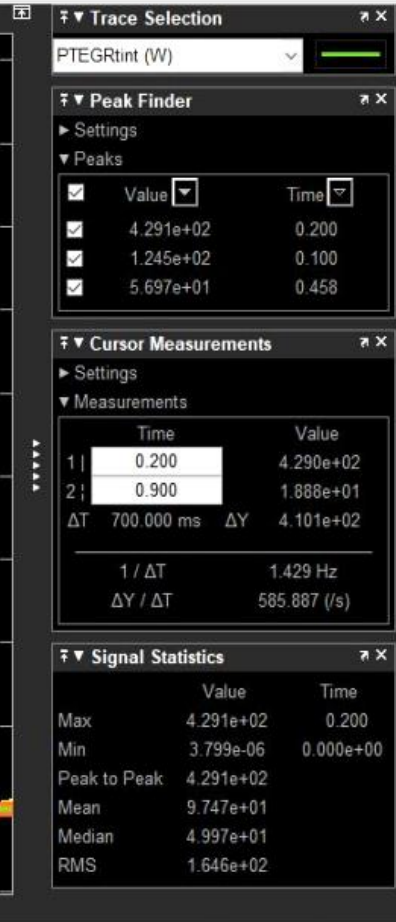

$\underline{\text { Resdy }}$

Sample based $T=1.100$

Figure 11d: 36 TEGs total internal resistance current, voltage and power losses dynamics; as the TEGs 10 configurations and temperatures auto change 
A Converter Input

File Tools View Simulation Help

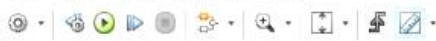

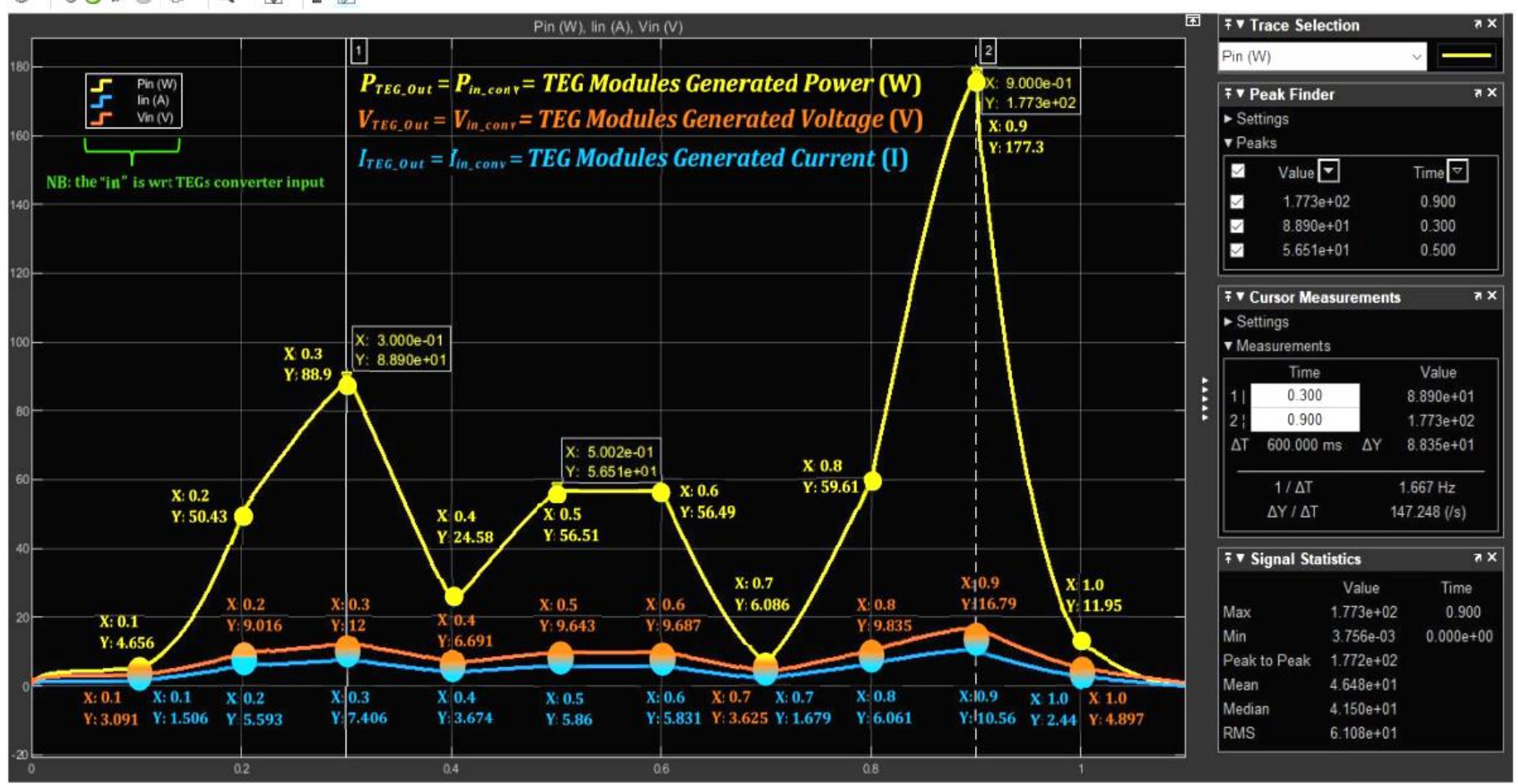

Figure 11e: 36 TEGs output power, voltage and current dynamics as the TEGs temperatures and 10 configurations auto change as simulation progresses

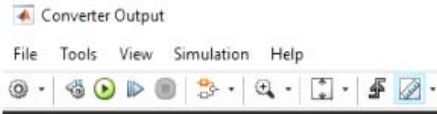

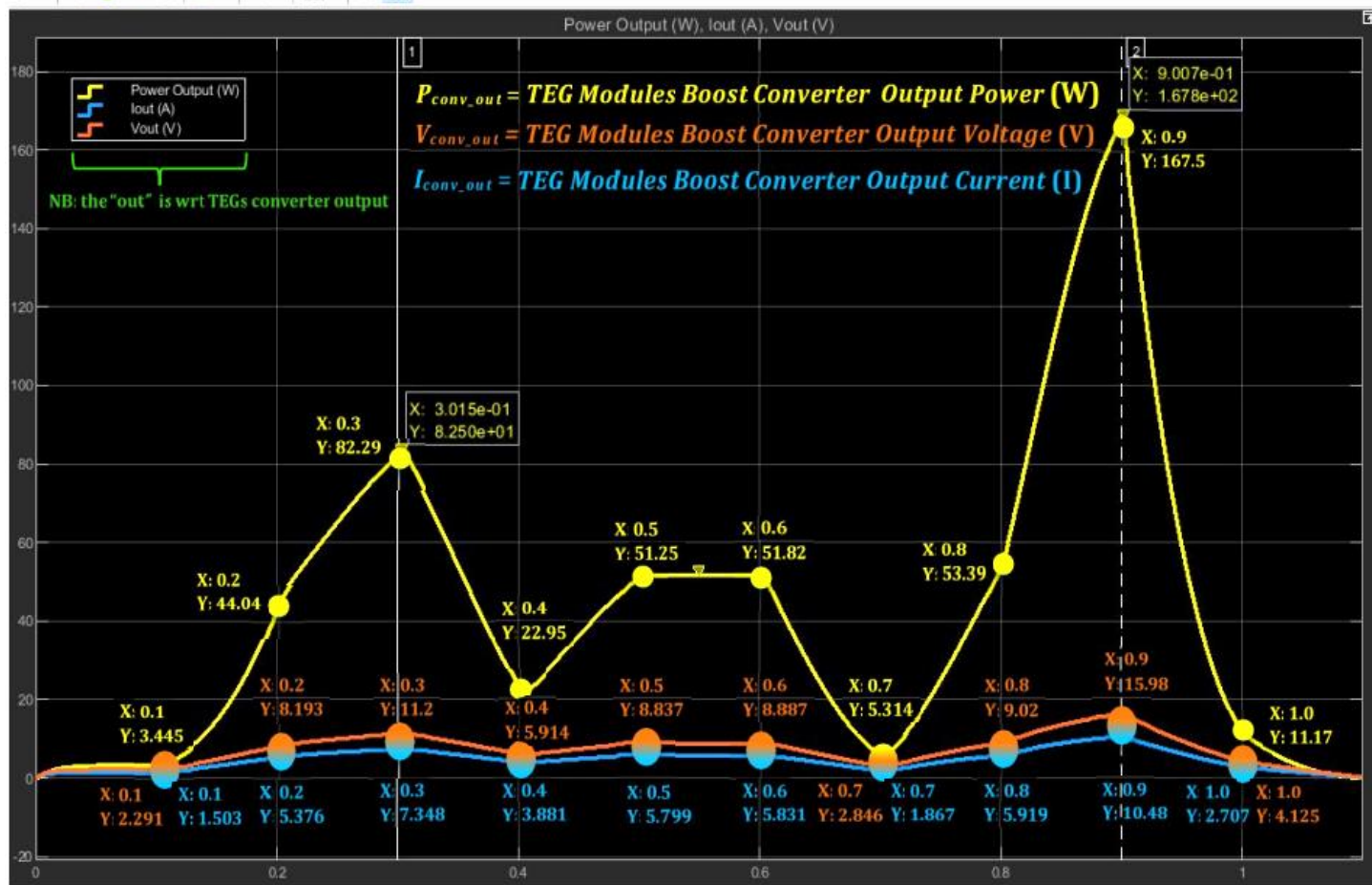

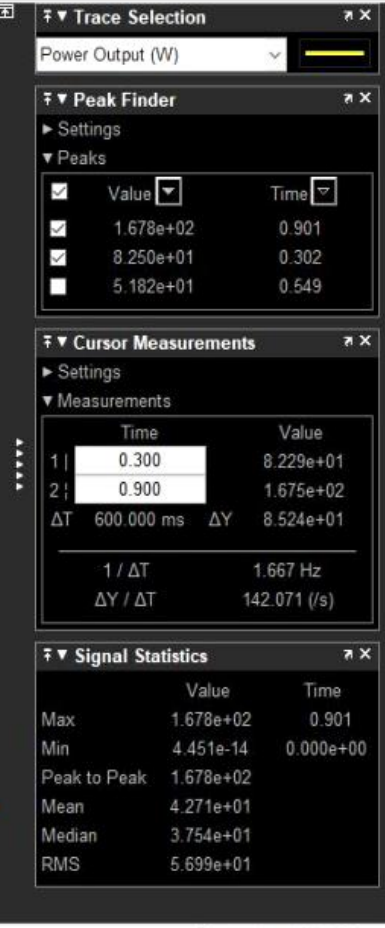

Sample based $T=1.100$

Figure 11f: 36 TEGs boost converter output power, voltage and current dynamics as the TEGs temperatures and the TEGs 10 configurations auto change 


\section{TEGs and TECs Simulations Results Discussions}

The TEGs/TECs simulations results demonstrated in Section 3 , are engaged below in their following respective sub-sections.

\subsection{TEGs Parameters Static Simulation Results Discussion}

Some of the crucial TEGs parameters simulated in Section 3.1. and the significance of the results are herein asserted. As exemplified in Figure 3, a TEGs generated power $P_{o}$ is proportional to its temperature difference $\Delta \mathrm{T}$ and output current $I$; however, $I$ above 5A (in this case) will decrease $P_{o}-$ which is because of the TEGs internal Ohmic heating as a result of the increasing output current $I$. The $\Delta \mathrm{T}, P_{o}$ and $I$ optimum operation points are emphasized in green in Figure 3. In Figure 4, a TEGs conversion efficiency $\eta$ is directly proportional to current output $I$ up to $\sim 5 \mathrm{~A}$ $\max$ (in this case) and decreases later as highlighted in green. It should be noted that $\eta$ is as well directly proportional to $P_{o}$. However, a TEG $P_{o}$ is reciprocally proportional to its p-n thermocouple junction resistance $r$ and as well to its total internal resistance $R_{t}$ (more than one connected TEG modules), though pro rata to $I$ up to $\sim 5 \mathrm{~A}$ (in this case) as portrayed in Figure 5. At this optimal point; $R_{t}$ or $R$ is $0 \Omega, I$ is $\sim 5 \mathrm{~A}$ maximum and $P_{o}$ is $\sim 105 \mathrm{~W}$ as highlighted in green. In Figure 6, the TEGs current output $I$ is proportional directly to the TEGs absorbed heat $Q_{h}$ (at temperature $T_{h}$ on the TEG hot-side) which in turn is directly dependent on the TEG $\Delta$ T. Figure 6 pictured the optimum point stressed-out in green. It should be noted that these results are not specific to a particular TEGs' connections - the results just fundamentally give a holistic theoretical understanding on what TEGs physical parameters must be taken into considerations, how they are interrelated, their associated dynamics and technical limitations and how they can be practically traded-off or optimized for optimal performance when designing TEGs power supply systems.

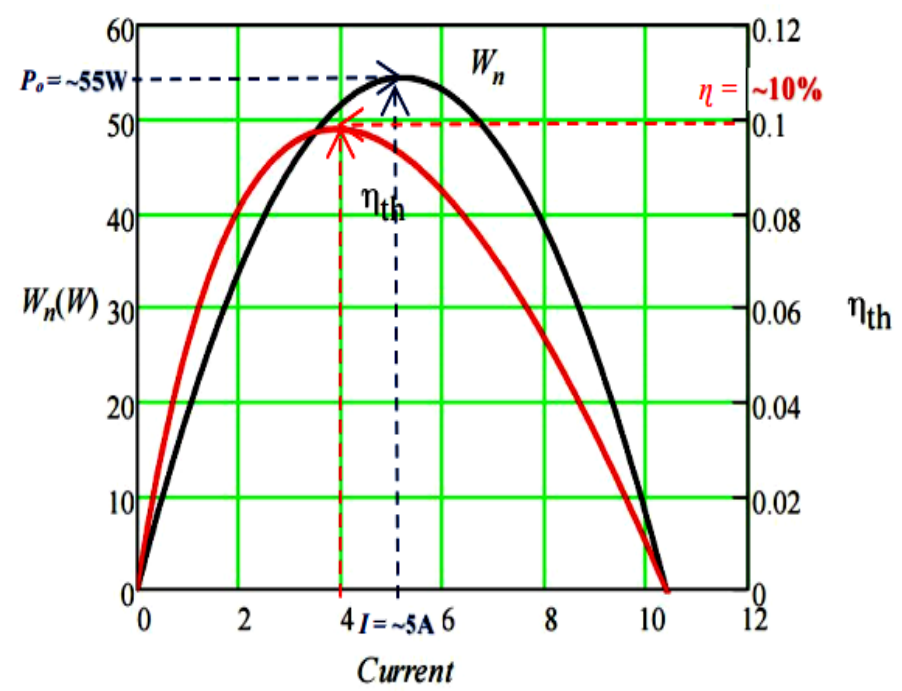

(A)

Figure 12: Validating our model with [9] - TEG (i) output power $P_{O}=\sim 55 \mathrm{~W}$ vs output current $I=\sim 5 \mathrm{~A}$ validating our Figure 3 result and (ii) conversion efficiency $\eta=\sim 10 \%$ vs output current $I=\sim 4 \mathrm{~A}$ validating our Figure 4 result.
Depicted in Figure 12, is a result of a typical TEG model simulated with Mathcad using TEG standard specifications from typical manufacturers data-sheet as presented in [9]. This was used as a benchmark to validate our TEG model simulation accuracy which is very close, besides a few discrepancies due to minor simulation settings differences. In light of this, our implemented TEG model can be used and developed further to simulate TEGs, including infinite series and parallel connections, which are central to our research and in large scale TEGs uses.

\subsection{TECs Parameters Static Simulation Results Discussion}

Some of the critical TECs parameters simulated in Section 3.2. and the importance of the results are herein articulated. Figure 7 illustrates that TECs $Q_{c}$ on TECs cold-side $T_{c}$, is reciprocally proportional to $\Delta \mathrm{T}$ but proportional directly to $I_{\text {in }}$ up to a maximum point, after which $Q_{c}$ starts dropping. The reasons are due to i) Joule heating (the more $I_{i n}$, the more the internal heating effect) and also ii) the second law of thermodynamics - simply put, heat flows from a hotter to a colder body; in this regards, the heating caused by the increasing $I_{\text {in }}$, increases the TECs internal temperature up to a temperature greater than that surrounding the TECs hot-side $T_{h}$; consequently, heat now starts to flow from the TECs hot-side to its cold-side, thus making the cooling process (heat pumping) on the TECs cold-side inefficient. In Figure 7 and highlighted in red, the $Q_{c}, \Delta \mathrm{T}$ and $I_{i n}$; display three optimal operation points depending on the TECs design constraints/ priorities. In option $1, Q_{c}$ is $115.677 \mathrm{~W}$ with a $\Delta \mathrm{T}$ of $1^{\circ} \mathrm{C}$ and $I_{i n}$ of $6 \mathrm{~A}$. In option $2, Q_{c}$ is $110.668 \mathrm{~W}$ with a $\Delta \mathrm{T}$ of $19^{\circ} \mathrm{C}$ and $I_{i n}$ of $14 \mathrm{~A}$. In option $3, Q_{c}$ is $105.664 \mathrm{~W}$ with a $\Delta \mathrm{T}$ of $4^{\circ} \mathrm{C}$ and $I_{\text {in }}$ of $16 \mathrm{~A}$. As evident, either $\Delta \mathrm{T}$ and or $I_{\text {in }}$ depending on the design constraints, can be optimized by either minimizing the TECs $\Delta \mathrm{T}$ and or maximizing TECs $I_{i n}$ to increase $Q_{c}$ within max operational limits. In Figure $8, P_{\text {in }}$ and $I_{\text {in }}$ are directly proportionally, which will initially increase $Q_{c}$ until a certain maximum limit, after which increasing $P_{\text {in }}$ and $I_{\text {in }}$ drop $Q_{c}$ - contrary to $\Delta \mathrm{T}$ which is inversely proportional to $Q_{c}$. The optimal operation point is highlighted in red. Figure 9 shows a TECs $P_{\text {in }}$ vs $I_{\text {in }}$ vs $R$. Normally $R$ is set fixed when designed by the manufacturer but now, with $R_{t}$ introduced, $R$ can be fairly altered and if it is matched to $R_{s}$, maximum power will be transferred to the TEC(s); thereby, optimizing $P_{\text {in }}$ and maximizing $Q_{c}$ as highlighted in red. Figure 10 demonstrates how $C o P$ akin to $Q_{c}$; increases with decreasing $\Delta \mathrm{T}$ and initially with increasing $I$ up to a maximum value and then starts decreasing, as current $I$ increases as shown variously in Figure 10. Depending on the design constraints, two optimal CoP operation points are evident as highlighted in red - in optimal operation point 1 , a $C o P$ of 3.3763 is achievable by minimizing $I_{\text {in }}$ to $1.8644 \mathrm{~A}$ and maximizing $\Delta \mathrm{T}$ to $9.322^{\circ} \mathrm{C}$; whereas in optimal operation point 2 , a CoP of 3.3638 is attainable by maximizing $I_{\text {in }}$ to $2.9831 \mathrm{~A}$ and minimizing $\Delta \mathrm{T}$ to $0^{\circ} \mathrm{C}$. Finally, our TECs model is reasonably validated by comparing a specific $Q_{c}$ of Figure 7 with that of Figure 13, as shown. The discrepancy is due to different TECs parameters setting. In sum, understanding the theory of TECs parameters and taking the various operational dynamics involved into considerations are very crucial in TEC(s) design/performance. 


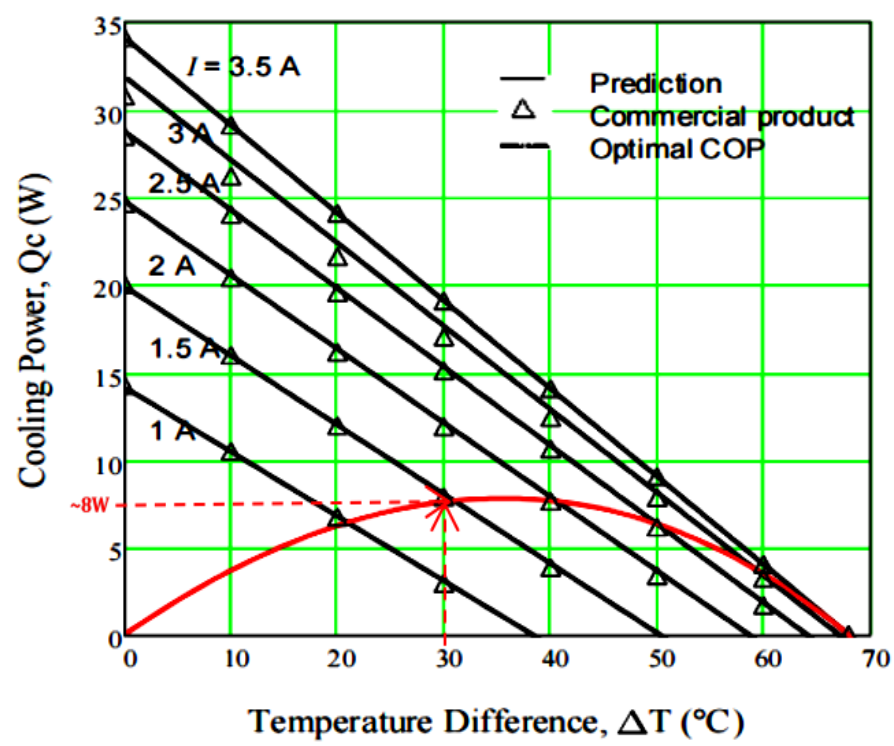

Figure 13: Validating our model with Lee, 2016 [9] - using TEC cooling power $Q_{c}$ $=\sim 8 \mathrm{~W}$ vs input current $I=\sim 1.5 \mathrm{~A}$ vs $\Delta \mathrm{T}=\sim 30^{\circ} \mathrm{C}$ to validate our TECs $Q_{c}$ in Figure 7 result with cooling power $Q_{c}=\sim 16 \mathrm{~W}$ vs input current $I=\sim 2 \mathrm{~A}$ vs $\Delta \mathrm{T}=\sim 30^{\circ} \mathrm{C}$.

\subsection{TEGs Dynamic Simulation Results Discussion}

Some of the critical TEGs dynamic simulated in Section 3.3. and the importance of the results are herein discussed. The TEGs temperatures and modules electrical connections (series, parallel, series/parallel) dynamics were simulated. In which beginning with the TEGs temperature dynamics, various arbitrary temperatures on the TEGs hot and cold sides as demonstrated in Figure $1 \mathrm{~b}$ and Figure 11a, as well as summarized in Table 1, were simply dynamically simulated using time-series inputs. As expected, the TEGs dynamically generated power, voltage and current; increased with increasing $T_{h}$ and $D T$ but with decreasing $T_{c}$.

Table 1: TEGs time-series inputs dynamic simulations results summary

\begin{tabular}{|c|c|c|c|c|c|c|c|c|c|c|}
\hline \multirow{2}{*}{ Parameters } & \multicolumn{10}{|c|}{ Matlab / Simulink Simulation Time } \\
\hline & 0.1 & 0.2 & 0.3 & 04 & 0.5 & 0.6 & & & & \\
\hline & \multicolumn{10}{|c|}{ TEG modules $T_{h}, T_{c}$ and $D T$ dynamic temperature inputs in ${ }^{\circ} \mathrm{C}$} \\
\hline & 60 & 120 & 125 & 75 & & & & 85 & & 150 \\
\hline & 10 & 20 & 25 & 30 & & & & 0 & & 45 \\
\hline$T E G$ & 50 & 100 & 100 & 45 & 65 & 65 & 25 & 85 & 195 & 105 \\
\hline Figur & \multicolumn{10}{|c|}{36 TEG modules in 10 dynamic $T_{s}, T_{p}, T_{t}$ and $R_{t}$ auto configuration } \\
\hline$T_{s}$ & 36 & 18 & 12 & 9 & 6 & 6 & 4 & 3 & 2 & 1 \\
\hline$T_{p}$ & 1 & 2 & 3 & 4 & 6 & 6 & 9 & 12 & 18 & 36 \\
\hline$T_{t}$ & 36 & 36 & 36 & 36 & 36 & 36 & 36 & 36 & 36 & 36 \\
\hline & & & & $3 .+24$ & & & & & & \\
\hline & \multicolumn{10}{|c|}{36 TEG modules ideal (if $T E G_{\text {Rtint }}=0$ ) power, voltage and current } \\
\hline $\operatorname{TEG} G_{P o c M}(\mathrm{~W})$ & \multirow{4}{*}{\multicolumn{10}{|c|}{\begin{tabular}{|cccccccc}
129.1479 .4423 .2 & 70.86 & 108.8 & 108.3 & 7.995 & 73.61 & 196.1 & 12.2 \\
85.7285 .7257 .15 & 19.29 & 18.57 & 18.57 & 4.763 & 12.14 & 18.57 & 5 \\
1.5065 .5937 .406 & 3.674 & 5.86 & 5.831 & 1.679 & 6.061 & 10.56 & 2.44 \\
36 TEG modules internal resistance power, voltage and current
\end{tabular}}} \\
\hline $\boldsymbol{T E} \boldsymbol{G}_{\text {Voc }}(\mathrm{V})$ & & & & & & & & & & \\
\hline$T E G_{I o c}$ & & & & & & & & & & \\
\hline & & & & & & & & & & \\
\hline TEG $G_{\text {Rtint }}(\Omega)$ & \multicolumn{10}{|c|}{54.8613 .726 .0963 .4291 .5241 .5240 .6770 .3810 .1690 .0423} \\
\hline PTEG Rtint $_{\text {(W) }}$ & \multicolumn{10}{|c|}{ 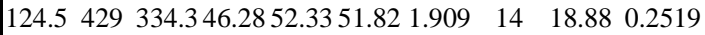 } \\
\hline$V T E G_{\text {Rtint }}(\mathrm{V})$ & \multicolumn{9}{|c|}{$82.6376 .7145 .15 \quad 12.6 \quad 8.93 \quad 8.887 \quad 1.1372 .3091 .788$} & 0.0103 \\
\hline ITEGRtint (A) & \multicolumn{9}{|c|}{$1.5065 .5937 .4063 .6745 .865 .8311 .6796 .061 \quad 10.56$} & 2.44 \\
\hline Figure $11 \mathrm{e}$ & \multicolumn{10}{|c|}{36 TEG modules generated (terminal) power, voltage and current } \\
\hline$P_{\text {TEG_Out }}(\mathrm{W})$ & \multicolumn{10}{|c|}{$4.65650 .4388 .924 .5856 .5156 .496 .08659 .61177 .3 \quad 11.95$} \\
\hline$V_{T E G \_O u t}(\mathrm{~V})$ & \multicolumn{9}{|c|}{$3.0919 .016 \quad 12 \quad 6.6919 .6439 .6873 .6259 .83516 .79$} & 4.897 \\
\hline ITEG_Out $(\mathrm{A})$ & \multirow{2}{*}{\multicolumn{9}{|c|}{$\begin{array}{llllll}1.5065 .5937 .4063 .674 & 5.86 & 5.831 & 1.679 & 6.061 & 10.56\end{array}$}} & 2.44 \\
\hline Figure 11f & \multicolumn{8}{|c|}{36 TEG modules boost converter output power, voltage and current } & & \\
\hline & \multirow{3}{*}{\multicolumn{10}{|c|}{ 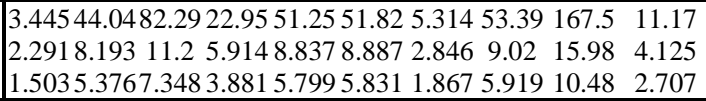 }} \\
\hline v_out $(\mathrm{V})$ & & & & & & & & & & \\
\hline$I_{\text {conv_out }}$ (A) & & & & & & & & & & \\
\hline
\end{tabular}

The TEG modules quantity used and most vitally in series, parallel and mixed connection were simulated, whereby as shown in Figure 1b and Figure 11b, as well as summarized in Table 1; 36 TEGs were arbitrary chosen and then arranged in 10 different combinations to study the effects of the various arrangements and when matched to a $1.524 \Omega$ electrical load. Each arrangement gives a different $R_{t}$, consequently giving different generated powers, voltages and currents. Figure 11c depicts the TEGs ideal power, voltage and current generated - assuming the TEGs $R_{t}$ or $T E G_{\text {Rtint }}$ is trivial. Figure 11d shows the power loss, voltage drop and Ohmic current due to the presence of TEG $G_{\text {Rtint }}$. Finally, Figures 11e and 11f, show the resultant output power, voltage and current supplied to the DC-DC boost converter and from it. As apparent, more TEG modules increased the output values; however, what is more insightful is how TEGs opt to be connected and matched to a $R_{L}-$ to ensure maximum power is transferred between $R_{t}$ and a $R_{L}$.

\section{Conclusions}

Sustainable energy is becoming popular to supplement the traditional grid and for private use, as well as for green economy. In view of this, we proffer thermoelectricity as an alternative energy source (TEGs) as well as an energy efficient load (TECs) for assorted applications that require low DC power, cooling and heating. However, TEG and TEC require multiple units connected in series and or in parallel to provide decent output and cooling powers respectively. Usually, the uninformed perception would be trying to utilize more TEGs and TECs with the hope to get more output and cooling powers respectively. However, our findings asserted this is not really the case, since i) TEG and TEC are not entirely linear devices, especially with increasing current, ii) TEG and TEC temperature difference $\Delta \mathrm{T}$ and current parameters have performance dynamics which must be operated within very strict optimal operation limits to guarantee efficiency and iii) TEGs and TECs total electrical resistance $R_{t}$ changes - increases when connected in series and decreases when connected in parallel. Thus, the overall power and efficiency will be affected, especially if the source and load resistances are not matched to transfer maximum power. In essence, our research major contributions include formulas developed for various TEGs/TECs parameters with focus on the TEG and TEC modules total resistance $R_{t}$ variations - when more than one TEG and or TEC modules are connected in infinite series and or in parallel combinations. Further contributions include detailed TEGs and TECs theoretical simulated models using Matlab/Simulink, whereby the TEGs and TECs models were used to easily simulate and investigate some thermoelectricity profound parameters performance dynamics, $R_{t}$ losses and to validate some of their operation points with industry standard models. Assorted large scale practical applications of TEGs and TECs were examined and in light of their results, our future work will include embarking on an actual lab design, testing our implemented models using them and refining ours accordingly while taking the physical dynamics into account. Thereafter, a practical pilot $1 \mathrm{~kW}$ implementation shall be devised for a low energy combined cooling, heating and power (CCHP) system - as an alternative energy green option for private use. 


\section{Nomenclature/Symbols}

A TEG/TEC $\mathrm{p}-\mathrm{n}$ junction thermocouple area in $\mathrm{m}^{2}$

CCHP Combined cooling, heating and power

$C F D \quad$ TEC(s) cold flux density in $\mathrm{W} / \mathrm{m}$

CoP TEC(s) coefficient of performance

$\mathrm{CoP}_{e} \quad \mathrm{TEC}(\mathrm{s}) \mathrm{CoP}$ expression

CoP $P_{\max }$ TECs maximum CoP

$\mathrm{CoP}_{\text {mid }} \quad \mathrm{TEC}(\mathrm{s})$ midpoint $\mathrm{CoP}$

$\mathrm{CoP}_{n} \quad \mathrm{TEC}(\mathrm{s})$ normalized CoP TEC(s)

$\Delta T \quad \mathrm{TEG}(\mathrm{s})$ temperature difference $\left(T_{h}-T_{c}\right)$ in ${ }^{\circ} \mathrm{C}$ or $\mathrm{K}$

$\Delta T_{\max } \quad \mathrm{TEC}(\mathrm{s})$ maximum temperature difference in ${ }^{\circ} \mathrm{C}$

$\Delta T_{n} \quad$ TEC(s) normalized temperature difference

$H F D$ TEG(s) heat flux density in $\mathrm{W} / \mathrm{m}^{2}$

I TEGs output current in ampere through the TEG(s)

$I_{\text {conv_out }}$ TEGs booster converter output current

$I_{\text {cop }} \quad$ TEC(s) current in ampere to yield CoP

Icp max $_{\text {max }}$

$I_{\text {in }}$

$\operatorname{Iin}_{n}$

$I_{\operatorname{Max}}$

$I_{\max }$

$I_{\text {mid }}$

$I_{n}$

ITEG $_{\text {Rtint }}$

$I_{T E G \_O u t}$

TEC(s) maximum cooling power current in ampere

TEC module(s) input current in ampere

TEC(s) normalized input current is the ratio of $I_{c o p}$ and $I_{\max }$

TEG(s) maximum output current in ampere

TEC(s) maximum input current in ampere when $Q_{c}$ $=0$

TEC(s) midpoint current in ampere

TEG(s) normalized output current

TEG ohmic current - results to TEG Ohmic or Joule heating

converter)

$K \quad$ TEC/TEG(s) thermal conductance in (W/K)

$k_{e} \quad \mathrm{TEG}(\mathrm{s}) / \mathrm{TEC}(\mathrm{s})$ effective thermal conductivity in $\mathrm{W} / \mathrm{mK}$

$L \quad$ TEG/TEC p-n junction thermocouple length in meter

$n \quad$ P-N thermocouples amount used in a TEG/TEC

$\eta \quad$ TEG(s) thermal/electrical/conversion efficiency

$\eta_{c} \quad$ Carnot efficiency

$\eta_{e} \quad$ TEG(s) conversion efficiency expression

$\eta_{n} \quad$ TEG(s) conversion efficiency normalized

$\eta_{m} \quad$ TEG(s) maximum conversion efficiency

$\eta_{m p} \quad$ TEGs max power conversion efficiency at the TEGs maximum $P_{o}$

$\rho \quad$ TEG/TEC electrical resistivity in $\Omega . \mathrm{m}$

$\rho_{e} \quad$ TEG(s)/TEC(s) effective electrical resistivity in $\Omega . \mathrm{m}$

$P_{\text {conv_out }}$

$P_{\text {in }}$

Pin $_{\text {mid }}$

Po

$P o_{\max }$

$P_{n}$

$P T E G_{\text {Rtint }}$

TEGs booster converter output power

TEC module(s) input power in watt

TEC(s) midpoint input power in watt

TEG(s) output power in watt - which is $Q_{h}-Q_{c}$

TEG(s) maximum output power in watt

TEG(s) normalized output power

$P_{T E G \_O u t}$

TEG generated power loss - due to TEG internal resistance

converter)

$Q_{c} \quad$ TEC module(s) cooling power on its cold-side in

(W)
$Q_{c}$

$Q_{h}$

$Q_{h}$

Qcp max

$Q c_{\max }$

$Q c_{\text {mid }}$

$Q c_{n}$

$r \quad$ TE device p-n thermocouples unit resistance in ohm

$R \quad$ TE device (TEG and TEC) module unit resistance in ohm

$R_{L} \quad$ TEGs electrical load resistance in $\Omega$ connected to the TEG(s)

$R_{s} \quad$ Power source resistance in ohm connected to the TECs

$R_{t} \quad$ TEG/TEC module(s) total resistance in ohms

$S \quad$ TE device Seebeck coefficient in V/K

Se TEG(s)/TEC(s) effective Seebeck coefficient in V/K

$\bar{T} \quad$ TE device average temperature $\left(T_{h}+T_{c}\right) / 2$ in $\mathrm{K}$ or ${ }^{\circ} \mathrm{C}$

$T_{c} \quad$ Temperature on TEG/TEC cold-side in ${ }^{\circ} \mathrm{C}$

$T_{h} \quad$ Temperature on TEG/TEC hot-side in ${ }^{\circ} \mathrm{C}$

TE Thermoelectric

TEC Thermoelectric cooler

TECsa TEC cold-side surface area

TEG Thermoelectric generator

$T E G_{\text {Ioc }}$ TEG ideal generated current - assuming there is no $T E G_{\text {Rtint }}$

$T E G_{P o c M}$ TEG ideal generated power - assuming there is no TEG Rtint

$T E G_{\text {Rtint }}$ TEG internal resistance $\left(R_{t}\right)$ - responsible for the power loss

TEGsa TEG hot-side surface area

TEGs $T_{c}$ TEGs cold side temperature

TEGs DT TEGs temperature difference

TEGs $T_{h}$ TEGs hot side temperature

$T E G_{V o c} \quad$ TEG ideal generated voltage - assuming there is no TEG $G_{\text {Rtint }}$

TEH Thermoelectric Energy Harvester

$T_{p} \quad$ TEGs/TECs module quantity connected in parallel

$T_{s} \quad$ TEGs/TECs module quantity connected in series

$T_{t} \quad$ TEG/TEC modules total quantity connected

$V_{\text {comvout }}$ TEGs booster converter output voltage

Vin $\quad$ TEC module(s) input voltage in volt

$V i n_{\max }$ TEC's max $V_{\text {in }}$ in $(\mathrm{V})$ that produces $\max \Delta T_{\max }$ when $I_{\text {in }}=I_{\text {max }}$

$\operatorname{Vin}_{n} \quad$ TEC(s) normalized input voltage is the ratio of $V_{\text {in }}$ and $\operatorname{Vin}_{\max }$

Vo TEG module(s) output voltage in volt

$V_{\text {max }} \quad$ TEG(s) maximum output voltage in volt

$V n \quad$ TEG(s) normalized output voltage

$V_{T E G_{-} O u t}$ TEGs generated voltage (input voltage to the boost converter)

$V T E G_{\text {Rtint }}$ TEG generated voltage drop - due to TEG internal resistance

WSN Wireless Sensors Network

$Z \quad$ TE device figure of merit in per $\mathrm{K}$

Ze TEG(s)/TEC(s) effective figure of merit in per $\mathrm{K}$ 


\section{$Z \bar{T} \quad$ TE device average dimensionless figure of merit}

\section{Acknowledgment}

This work was supported in parts by the Cape Peninsula University of Technology CPGS and the University of the Western Cape HySA Systems.

\section{Data availability}

Research in progress - data available upon completion.

\section{Conflict of Interest}

Authors declare no conflict of interest.

\section{References}

[1] M.L. van der Walt, J. van den Berg, M. Cameron, "State of Renewable Energy in South Africa", South Africa Department of Energy, Pretoria, 2017, http://www.energy.gov.za/files/media/Pub/2017-State-of-ReewableEnergy-in-South-Africa.pdf.

[2] N.P. Bayendang, M.T. Kahn, V. Balyan, "A structural review of thermoelectricity for fuel cells CCHP applications," Hindawi Journal of Energy 2020, 1-23, 2020, https://doi.org/10.1155/2020/2760140/

[3] Y.-S. Noh, J.-I. Seo, W.-J. Choi, J.-H. Kim, H.V. Phuoc, H.-S. Kim, S.-G. Lee, "17.6 A Re-configurable DC-DC Converter for Maximum TEG Energy Harvesting in a Battery-Powered Wireless Sensor Node," 2021 IEEE International Solid-State Circuits Conference (ISSCC),San Francisco, CA, USA, 266-268, 2021, doi: 10.1109/ISSCC42613.2021.9365811.

[4] D. Charris, D. Gómez, M. Pardo, "A Portable Thermoelectric Energy Harvesting Unit to Power Up Outdoor Sensors and Devices," 2019 IEEE Sensors Applications Symposium (SAS), Sophia Antipolis, France, 1-6, 2019, doi: 10.1109/SAS.2019.8705985.

[5] J. Singh, P. Kuchroo, H. Bhatia, E. Sidhu, "Floating TEG based solar energy harvesting system," 2016 International Conference on Automatic Control and Dynamic Optimization Techniques (ICACDOT), Pune, India, 763-766, 2016, doi: 10.1109/ICACDOT.2016.7877689.

[6] Q. Wan, Y. Teh, P.K.T. Mok, "Analysis of a Re-configurable TEG Array for High Efficiency Thermoelectric Energy Harvesting," 2016 IEEE Asia Pacific Conference on Circuits and Systems (APCCAS), Jeju, Korea (South), 662-665, 2016, doi: 10.1109/APCCAS.2016.7804084

[7] R. Chein, G. Huang, "Thermoelectric cooler application in electronic cooling", Applied Thermal Engineering, 24(14-15), 2207-2217, 2004, https://doi.org/10.1016/j.applthermaleng.2004.03.001.

[8] I.R. Belovski, A.T. Aleksandrov, "Examination of the Characteristics of a Thermoelectric Cooler in Cascade," 2019 X National Conference with International Participation (ELECTRONICA), 1-4, 2019, doi: 10.1109/ELECTRONICA.2019.8825631.

[9] H. Lee, Thermoelectrics: design and materials, John Wiley \& Sons, Inc., Wiley, New Jersey, USA, 2016.

[10] H. Mamur, Y. Coban, "Detailed Modeling of a Thermoelectric Generator for Maximum Power Point Tracking", Turkish Journal of Electrical Engineering \& Computer Sciences, 28, 124-139, 2020, https://doi.org/10.3906/elk-1907166.

[11] N.P. Bayendang, M.T. Kahn, V. Balyan, I. Draganov, S. Pasupathi, "A Comprehensive Thermoelectric Generator (TEG) Modelling", AIUE Congress 2020: Energy and Human Habitat Conference, Cape Town, South Africa, 1-7, 2020; Publisher Zenodo: Geneva, Switzerland, Available online 2020, http://doi.org/10.5281/zenodo.4289574.

[12] N.P. Bayendang, M.T. Kahn, V. Balyan, I. Draganov, S. Pasupathi, "A Comprehensive Thermoelectric Cooler (TEC) Modelling", AIUE Congress 2020: International Conference on Use of Energy, Cape Town, South Africa, 1-7, 2020; Publisher SSRN: Rochester, NY, USA, Available online 2021, https://ssrn.com/abstract $=3735378$ http://dx.doi.org/10.2139/ssrn.3735378.

[13] F. Felgner, L. Exel, M. Nesarajah, G. Frey, "Component-oriented modeling of thermoelectric devices for energy system design," in IEEE Transactions on Industrial Electronics, 61(3), 1301-1310, 2014, doi: 10.1109/TIE.2013.2261037.
[14] C. Luo, R. Wang, W. Yu, W. Zhou, "Parametric study of a thermoelectric module used for both power generation and cooling," Renewable Energy, 154, 542-552, 2020, https://doi.org/10.1016/j.renene.2020.03.045.

[15] C. Liu, P. Chen, K. Li. "A $1 \mathrm{~kW}$ Thermoelectric Generator for Lowtemperature Geothermal Resources," PROCEEDINGS, 39 ${ }^{\text {th }}$ Workshop on Geothermal Reservoir Engineering, Stanford University, Stanford, California, USA, 24-26, 2014, SGP-TR-202. https://pangea.stanford.edu/ERE/pdf/IGAstandard/SGW/2014/Li.pdf [Date accessed: August 3, 2021].

[16] S.O. Giwa, C.N. Nwaokocha, A.T. Layeni, O.O. Olaluwoye, "Energy harvesting from household heat sources using a thermoelectric generator module," Nigerian Journal of Technological Development, 16(3), 2019, http://dx.doi.org/10.4314/njtd.v16i3.6.

[17] M.W. Aljibory, H.T. Hashim, W.N. Abbas, "A Review of Solar Energy Harvesting Utilizing a Photovoltaic-Thermoelectric Integrated Hybrid System," IOP Conference Series: Materials Science and Engineering, $4^{\text {th }}$ International Conference on Engineering Sciences (ICES 2020), 1067, 2021, Kerbala, Iraq, doi:10.1088/1757-899X/1067/1/012115.

[18] X. Hu, C. Jiang, X. Fan, B. Feng, P. Liu, Y. Zhang, R. Li, Z. He, G. Li, Y. $\mathrm{Li}$, "Investigation on waste heat recovery of a nearly kilowatt class thermoelectric generation system mainly based on radiation heat transfer," Energy Sources, Part A: Recovery, Utilization, and Environmental Effects, 2020, https://doi.org/10.1080/15567036.2020.1829190.

[19] M.Y. Fauzan, S.M. Muyeen, S. Islam, "Experimental modelling of grid-tied thermoelectric generator from incinerator waste heat," International Journal of Smart Grid and Clean Energy, 2020, doi: 10.12720/sgce.9.2.304-313.

[20] M. Ebrahimi, E. Derakhshan, "Design and evaluation of a micro combined cooling heating and power system based on polymer exchange membrane fuel cell and thermoelectric cooler", Energy Conversion and Management, 171, 507-517, 2018, https://doi.org/10.1016/j.enconman.2018.06.007.

[21] A.G. Rösch, A. Gall, S. Aslan, M. Hecht, L. Franke, M.M. Mallick, L. Penth, D. Bahro, D. Friderich, U. Lemmer, "Fully printed origami thermoelectric generators for energy-harvesting," NPJ Flex Electrons, 5(1), 2021, https://doi.org/10.1038/s41528-020-00098-1.

[22] V.B. Abhijith, K. Narayanaswamy, C.M. Pooja, S.V. Prasad, R. Sambhu, "Household Application of Thermoelectric Generator in the Field of Propagating Power from Waste Heat," AIP Conference Proceedings 2297, 020010, 2020, https://doi.org/10.1063/5.0031699.

[23] F. Afshari, "Experimental and numerical investigation on thermoelectric coolers for comparing air-to-water to air-to-air refrigerators," Journal of Thermal Analysis and Calorimetry, 144, 855-868, 2021, https://doi.org/10.1007/s10973-020-09500-6. 